\title{
Additive semisimple multivariable codes over $\mathbb{F}_{4}$
}

\author{
E. Martínez-Moro • A. Piñera-Nicolás • I.F. \\ Rúa
}

the date of receipt and acceptance should be inserted later

\begin{abstract}
The structure of additive multivariable codes over $\mathbb{F}_{4}$ (the Galois field with 4 elements) is presented. The semisimple case (i.e., when the defining polynomials of the code have no repeated roots) is specifically addressed. These codes extend in a natural way the abelian codes, of which additive cyclic codes of odd length are a particular case. Duality of these codes is also studied.
\end{abstract}

Keywords Additive multivariable codes, abelian codes, quantum codes, duality

Mathematics Subject Classification (2000) 11T61, 94B99, 81P70, 13M10

\section{Introduction}

Quantum codes are designed to detect and correct the errors produced in quantum computations $[14,15]$. These codes can be constructed with the help of specific classical codes, called additive, over $\mathbb{F}_{4}$ (the Galois field with 4 elements) [2]. An additive code of length $n$ is a subgroup of $\mathbb{F}_{4}^{n}$ under addition. The particular case of additive cyclic codes has been considered in [5]. An additive code $\mathcal{C}$ is called cyclic if, whenever $c=\left(c_{1}, \ldots, c_{n}\right) \in C$, then its cyclic shift $\left(c_{2}, \ldots, c_{n}, c_{1}\right)$ is also a codeword in $\mathcal{C}$. These codes are related to properties of the ring $\mathbb{F}_{4}[X] /\left\langle X^{n}-1\right\rangle$. In the case $n$ odd, the semisimple structure of this ring can be used to obtain a complete description of the codes [7]. The case $n$ even has been also considered [8].

Many authors have stated that many classical codes are ideals in certain algebras over a finite field, see for example $[1,3,12]$. In particular, multivariable codes have been considered, i.e., codes that can be viewed as ideals of the quotient ring $\mathbb{F}_{4}\left[X_{1}, \ldots, X_{r}\right] /\left\langle t_{1}\left(X_{1}\right), \ldots, t_{r}\left(X_{r}\right)\right\rangle$ (where $t_{i}\left(X_{i}\right) \in \mathbb{F}_{4}\left[X_{i}\right]$ are fixed polynomials). Abelian codes, i.e., multivariable codes where $t_{i}\left(X_{i}\right)=X_{i}^{n_{i}}-1$, for all $1 \leq i \leq r$, are particular cases, and they extend classical cyclic codes. These types of codes

E. Martínez-Moro · A. Piñera-Nicolás

Institute of Mathematics (IMUVa) and Applied Mathematics Department, Universidad de Valladolid. E-mail: edgar@maf.uva.es, anicolas@maf.uva.es

I.F. Rúa

Departamento de Matemáticas, Universidad de Oviedo. E-mail: rua@uniovi.es 
have been also constructed if the underlying ring is not a field, but a finite chain ring $[9,10]$.

In this paper we describe additive multivariable codes over the finite field $\mathbb{F}_{4}$, when the polynomials $t_{i}\left(X_{i}\right) \in \mathbb{F}_{2}\left[X_{i}\right]$ have no repeated-roots. The semisimple structure of the rings $\mathcal{A}_{4}=\mathbb{F}_{4}\left[X_{1}, \ldots, X_{r}\right] /\left\langle t_{1}\left(X_{1}\right), \ldots, t_{r}\left(X_{r}\right)\right\rangle$ and $\mathcal{A}_{2}=$ $\mathbb{F}_{2}\left[X_{1}, \ldots, X_{r}\right] /\left\langle t_{1}\left(X_{1}\right), \ldots, t_{r}\left(X_{r}\right)\right\rangle$ is fundamental in this description.

The paper is organized as follows. In Section 2 we review the basic terminology and the results concerning the decomposition of the rings $\mathcal{A}_{4}$ and $\mathcal{A}_{2}$, and their relation. Section 3 is devoted to the description of the structure of the additive codes. In Section 4 we study the duals of abelian semisimple codes. Finally in Section 5 we characterize those non-trivial abelian semisimple codes that are selfdual.

\section{Preliminaries}

In this section we will obtain the structure of the ambient space of additive semisimple multivariable codes over the finite field $\mathbb{F}_{4}$. That is, we will describe explicitly the structure of the $\operatorname{ring} \mathcal{A}_{4}=\mathbb{F}_{4}\left[X_{1}, \ldots, X_{r}\right] /\left\langle t_{1}\left(X_{1}\right), \ldots, t_{r}\left(X_{r}\right)\right\rangle$, where $t_{i}\left(X_{i}\right) \in \mathbb{F}_{2}\left[X_{i}\right]$ for all $i=1, \ldots, r$. In order to obtain this description we will decompose this ring as a direct sum of ideals. See [13] for proofs and details about this decomposition.

2.1 Decomposition of $\mathbb{F}_{q}\left[X_{1}, \ldots, X_{r}\right] /\left\langle t_{1}\left(X_{1}\right), \ldots, t_{r}\left(X_{r}\right)\right\rangle$

Let $q=p^{e}(p$ prime $)$, and let $I=\left\langle t_{1}\left(X_{1}\right), \ldots, t_{r}\left(X_{r}\right)\right\rangle \triangleleft \mathbb{F}_{q}\left[X_{1}, \ldots, X_{r}\right]$ be the ideal generated by monic polynomials $t_{i}\left(X_{i}\right) \in \mathbb{F}_{p}\left[X_{i}\right]$, of degree $n_{i}, i=1, \ldots, r$ (in this paper we are concerned with the case $\left.q=2^{2}\right)$. Let $H_{i}$ be the set of roots of $t_{i}\left(X_{i}\right)$ in an suitable extension field of $\mathbb{F}_{q}$ for each $i=1, \ldots, r$. We require that $t_{i}\left(X_{i}\right)$ has no multiple roots for all $i=1, \ldots, r$. We are interested in the decomposition of the algebra $\mathcal{A}_{q}=\mathbb{F}_{q}\left[X_{1}, \ldots, X_{r}\right] /\left\langle t_{1}\left(X_{1}\right), \ldots, t_{r}\left(X_{r}\right)\right\rangle$.

Definition 1 Let $\mu=\left(\mu_{1}, \ldots, \mu_{r}\right) \in H_{1} \times \ldots \times H_{r}$, then we define the $q$-class of $\mu$ as

$$
C_{q}(\mu)=\left\{\left(\mu_{1}^{q^{s}}, \ldots, \mu_{r}^{q^{s}}\right) \mid s \in \mathbb{N}\right\}
$$

Proposition 1 Let $\mu=\left(\mu_{1}, \ldots, \mu_{r}\right) \in H_{1} \times \ldots \times H_{r}$ and let $q_{i}$ be the degree of the minimal polynomial of $\mu_{i}$ over $\mathbb{F}_{q}$ for each $i=1, \ldots, r$. Then we have that

1. $\left|C_{q}(\mu)\right|=$ l.c.m. $\left(q_{1}, q_{2}, \ldots, q_{r}\right)=\left[\mathbb{F}_{q}\left(\mu_{1}, \ldots, \mu_{r}\right): \mathbb{F}_{q}\right]$.

2. The set $\mathcal{C}_{q}$ of $q$-classes $C_{q}(\mu)$ is a partition of $H_{1} \times \ldots \times H_{r}$.

3. For each ideal $\mathcal{I} \triangleleft \mathbb{F}_{q}\left[X_{1}, \ldots, X_{r}\right] / I$ the affine variety $V(\mathcal{I})$ of common zeros of the elements in $\mathcal{I}$ is a union of classes.

Definition 2 Let $K$ be an algebraic extension of $\mathbb{F}_{q}$ and let $\alpha \in K$. Let us denote by $\operatorname{Irr}\left(\alpha, \mathbb{F}_{q}\right)$ the minimal polynomial of $\alpha$ over the field $\mathbb{F}_{q}$. If $\mu=\left(\mu_{1}, \ldots, \mu_{r}\right) \in$ $H_{1} \times \ldots \times H_{r}$, then consider the following polynomials:

1. $p_{\mu, i}\left(X_{i}\right)=\operatorname{Irr}\left(\mu_{i}, \mathbb{F}_{q}\right)$, and $d_{\mu, i}=\operatorname{deg} p_{\mu, i}$ for all $i=1, \ldots, r$.

2. $w_{\mu, i}\left(\mu_{1}, \ldots, \mu_{i-1}, X_{i}\right)=\operatorname{Irr}\left(\mu_{i}, \mathbb{F}_{q}\left(\mu_{1}, \ldots, \mu_{i-1}\right)\right)$ for all $i=2, \ldots, r$. 
3. $\pi_{\mu, i}\left(\mu_{1}, \ldots, \mu_{i-1}, X_{i}\right)=p_{\mu, i}\left(X_{i}\right) / w_{\mu, i}\left(\mu_{1}, \ldots, \mu_{i-1}, X_{i}\right)$ for all $i=2, \ldots, r$.

Remark 1 All the polynomials in the definition above can be seen as polynomials in $\mathbb{F}_{q}\left[X_{1}, \ldots, X_{r}\right]$ (substituting $\mu_{i}$ by $X_{i}$ ) and the following ring isomorphism holds

$$
\mathbb{F}_{q}\left[X_{1}, \ldots, X_{r}\right] /\left\langle p_{\mu, 1}, w_{\mu, 2}, \ldots, w_{\mu, r}\right\rangle \cong \mathbb{F}_{q}\left(\mu_{1}, \ldots, \mu_{r}\right) .
$$

Moreover, if $\mu^{\prime} \in C_{q}(\mu)$, then $p_{\mu, i}=p_{\mu^{\prime}, i} i=1, \ldots, r$ and $w_{\mu, i}=w_{\mu^{\prime}, i}, \pi_{\mu, i}=$ $\pi_{\mu^{\prime}, i} i=2, \ldots, r$. Thus, if $C=C_{q}(\mu)$ is the $q$-class of $\mu$, we will write $p_{C, i}=$ $p_{\mu, i}, d_{C, i}=d_{\mu, i}, w_{C, i}=w_{\mu, i}, \pi_{C, i}=\pi_{\mu, i}$. Analogously, the ideal $\left\langle p_{C, 1}, w_{C, 2}, \ldots, w_{C, r}\right\rangle$ will be denoted by $I_{C}$.

Definition 3 Let $\mu=\left(\mu_{1}, \ldots, \mu_{r}\right) \in H_{1} \times \ldots \times H_{r}$. If $C=C_{q}(\mu)$ is the $q$-class of $\mu$, we define the following polynomial in $\mathbb{F}_{q}\left[X_{1}, \ldots, X_{r}\right]$

$$
h_{C}\left(X_{1}, \ldots, X_{r}\right)=\prod_{i=1}^{r} \frac{t_{i}\left(X_{i}\right)}{p_{C, i}\left(X_{i}\right)} \prod_{i=2}^{r} \pi_{C, i}\left(X_{i}, \ldots, X_{r}\right) .
$$

Proposition 2 Let $\mu=\left(\mu_{1}, \ldots, \mu_{r}\right) \in H_{1} \times \ldots \times H_{r}$. If $C=C_{q}(\mu)$ is the $q-$ class of $\mu$, then

1. The annihilator of $\left\langle h_{C}+I\right\rangle$ (in $\left.\mathcal{A}_{q}\right)$ is $I_{C}+I$,

2. The set of zeros of $h_{C}$ is $H_{1} \times \ldots \times H_{r} \backslash C$,

3. The set of zeros of $I_{C}$ is $C$.

\section{Theorem 1 (Decomposition of the ambient space $\mathcal{A}_{q}$ )}

$$
\mathcal{A}_{q}=\mathbb{F}_{q}\left[X_{1}, \ldots, X_{r}\right] / I \cong \bigoplus_{C \in \mathcal{C}_{q}}\left\langle h_{C}+I\right\rangle
$$

where $\left\langle h_{C}+I\right\rangle \cong \mathbb{F}_{q}\left[X_{1}, \ldots, X_{r}\right] / I_{C}$ is a finite field isomorphic to $\mathbb{F}_{q}\left(\mu_{1}, \ldots, \mu_{r}\right) \cong$ $\mathbb{F}_{q|C|}$, and so the algebra $\mathcal{A}_{q}$ is semisimple. Hence, there exists a unique set of primitive orthogonal idempotents $\left\{e_{C}+I\right\}_{C \in \mathcal{C}_{q}} \subseteq \mathcal{A}_{q}$ such that $1+I=\sum_{C \in \mathcal{C}_{q}} e_{C}+I$ and $\mathcal{A}_{q}\left(e_{C}+I\right) \cong\left\langle h_{C}+I\right\rangle$. Namely, the idempotent $e_{C}+I$ is exactly the element $g_{C} h_{C}+I$, with $g_{C} h_{C}+I_{C}=1+I_{C}$, and its set of zeros is $H_{1} \times \ldots \times H_{r} \backslash C$

Remark 2 Notice that if $q=4, r=1$ and $t_{1}(X)=X^{n}-1$ with $n$ odd, we obtain the ambient space of additive cyclic codes over $\mathbb{F}_{4}$ described in [7]. In this case, if $\alpha$ is a primitive $n t h$-root of unity and $\mu=\alpha^{i}$, then the exponents of the elements in the $q$-class $C=C_{4}(\mu)$ are the elements of the 4-cyclotomic coset containing $i$. Also, each direct summand $h_{C}+I \cong \mathbb{F}_{4^{\lambda} C}$, where $\lambda_{C}$ is the size of the $q$-class $C$, that is, the degree of the irreducible polynomial $p_{C}$.

Classical multivariable codes over $\mathbb{F}_{4}$ are defined as the ideals of the algebra $\mathcal{A}_{4}$. However, since additive codes are no longer closed under multiplication by arbitrary elements of $\mathbb{F}_{4}$, they do not correspond to ideals of this ring. This type of codes are related instead to submodules of $\mathcal{A}_{4}$, when viewed as a module over one of its subrings.

Lemma 1 If $f \in \mathbb{F}_{q}\left[X_{1}, \ldots, X_{r}\right]$, then there exists a unique polynomial $N F(f) \in$ $\mathbb{F}_{q}\left[X_{1}, \ldots, X_{r}\right]$ such that $f+I=N F(f)+I$, and $\operatorname{deg}_{X_{i}}(N F(f))<n_{i}$. It is called the normal form of $f$ w.r.t. $I$. Moreover, two polynomials $f, g \in \mathbb{F}_{q}\left[X_{1}, \ldots, X_{r}\right]$ satisfy $f+I=g+I$ if and only if $N F(f)=N F(g)$. In particular, all classes $f+I$, where $0 \neq f$ and $\operatorname{deg}_{X_{i}}(N F(f))<n_{i}$, for all $1 \leq i \leq r$, are not zero. 
Proof Consider the lexicographic monomial order with $X_{1}>X_{2}>\cdots>X_{r}$. Then, $\left\{t_{1}\left(X_{1}\right), \ldots, t_{r}\left(X_{r}\right)\right\}$ is a Groebner basis of $I$ (actually its reduced Groebner basis w.r.t. such an order), and so the result follows from [4, Chapter 2, Section 9, Theorem 3 and Proposition 4].

Definition 4 Let $S$ be the set of elements $f+I \in \mathcal{A}_{q}$ such that $N F(f) \in \mathbb{F}_{p}\left[X_{1}, \ldots, X_{r}\right]$.

¿From now on, let us denote by $J$ the ideal in $\mathbb{F}_{p}\left[X_{1}, \ldots, X_{r}\right]$ generated by $t_{i}\left(X_{i}\right), i=1, \ldots, r$, and by $\mathcal{A}_{p}$ the algebra $\mathbb{F}_{p}\left[X_{1}, \ldots, X_{r}\right] / J$ (notice that $t_{i}\left(X_{i}\right) \in$ $\mathbb{F}_{p}\left[X_{i}\right]$, for all $\left.i=1, \ldots, r\right)$.

Proposition 3 There exists a ring monomorphism $\varphi: \mathcal{A}_{p} \rightarrow \mathcal{A}_{q}$ such that $\varphi(f+J)=$ $f+I$, for all $f \in \mathbb{F}_{p}\left[X_{1}, \ldots, X_{r}\right]$. The set $S$ is the image of this map, and so it is a subring of $\mathcal{A}_{q}$ isomorphic to $\mathcal{A}_{p}$.

Proof Consider the ring homomorphisms $\pi_{q} \circ i: \mathbb{F}_{p}\left[X_{1}, \ldots, X_{r}\right] \rightarrow \mathcal{A}_{q}$, given by $\left(\pi_{q} \circ i\right)(f)=f+I$, and $\pi_{p}: \mathbb{F}_{p}\left[X_{1}, \ldots, X_{r}\right] \rightarrow \mathcal{A}_{p}$, given by $\pi_{p}(f)=f+J$. Since $\operatorname{ker} \pi_{p} \subseteq \operatorname{ker} \pi_{q} \circ i$, there exists a ring homomorphism $\varphi: \mathcal{A}_{p} \rightarrow \mathcal{A}_{q}$ such that $\varphi(f+\bar{J})=f+I$, for all $f \in \mathbb{F}_{p}\left[X_{1}, \ldots, X_{r}\right]$.

For all $f \notin J$, we have that $f+J=N F(f)+J$, where $0 \neq N F(f) \in$ $\mathbb{F}_{p}\left[X_{1}, \ldots, X_{r}\right] \subseteq \mathbb{F}_{q}\left[X_{1}, \ldots, X_{r}\right]$, and $\operatorname{deg}_{X_{i}}(N F(f))<n_{i}$. So, $\varphi(f+J)=\varphi(N F(f)+$ $J)=N F(f)+I \neq I$, and the map is injective. Since $\operatorname{Im}(\varphi) \subseteq S$, a simple counting argument let us conclude the equality $\operatorname{Im}(\varphi)=S$, and so $\mathcal{A}_{p} \cong S$.

Definition 5 An additive (semisimple) multivariable code over $\mathbb{F}_{q}$ is a submodule of the module ${ }_{S} \mathcal{A}_{q}$.

Remark 3 These codes are called semisimple because the roots of the polynomials $t_{i}\left(X_{i}\right)$ are required to be simple. If $q=4, r=1, t_{1}(X)=X^{n}-1$, with $n$ odd, additive multivariable codes over $\mathbb{F}_{4}$ are exactly the additive cyclic codes over $\mathbb{F}_{4}$ described in [7].

Remark 4 It is just a straight forward fact that a semisimple code on $\mathcal{A}_{2}$ can be seen as a shortened code of an abelian code choosing adequate polynomials $X_{i}^{n_{i}}-1$ such that $t_{i}\left(X_{i}\right) \mid X_{i}^{n_{i}}-1$ (thus we must shorten the codes in the positions not in $\left.t_{i}\right)$. This follows directly from the fact that the ideals of $\mathbb{F}[X] /\langle t(X)\rangle$ ( $t$ having simple roots) are shortened cyclic codes (see for example [11, Section 8.10]).

Example 1 We shall illustrate the contents of the paper with the help of the following running example. Let us consider $t_{1}\left(X_{1}\right)=X_{1}^{7}+1, t_{2}\left(X_{2}\right)=X_{2}^{3}+1$ polynomials in $\mathbb{F}_{q}\left[X_{1}, X_{2}\right]$. The normal form of an element $f+I$, which has the shape $\sum_{j=0}^{6} \sum_{i=0}^{2} f_{i j} X_{1}^{j} X_{2}^{i}$, will be written as the matrix

$$
F=\left(f_{i j}\right)=\left[\begin{array}{llll}
f_{00} & f_{01} & \ldots & f_{06} \\
f_{10} & f_{11} & \ldots & f_{16} \\
f_{20} & f_{21} & \ldots & f_{26}
\end{array}\right]
$$

We shall describe the decompositions of the algebras $\mathcal{A}_{q}$ for $q=2$ and 4 . 
- If $q=2$, then the set of 2 -classes is the following one:

$$
\begin{aligned}
\mathcal{C}_{2}=\{ & \{(1,1)\},\left\{(\mu, 1),\left(\mu^{2}, 1\right),\left(\mu^{4}, 1\right)\right\},\left\{\left(\mu^{6}, 1\right),\left(\mu^{5}, 1\right),\left(\mu^{3}, 1\right)\right\}, \\
& \left\{(1, \omega),\left(1, \omega^{2}\right)\right\},\left\{(\mu, \omega),\left(\mu^{2}, \omega\right),\left(\mu^{4}, \omega\right),\left(\mu, \omega^{2}\right),\left(\mu^{2}, \omega^{2}\right),\left(\mu^{4}, \omega^{2}\right)\right\}, \\
& \left.\left\{\left(\mu^{6}, \omega\right),\left(\mu^{5}, \omega\right),\left(\mu^{3}, \omega\right),\left(\mu^{6}, \omega^{2}\right),\left(\mu^{5}, \omega^{2}\right),\left(\mu^{3}, \omega^{2}\right)\right\}\right\}
\end{aligned}
$$

where $\mu \in \mathbb{F}_{2^{3}}, \omega \in \mathbb{F}_{2^{2}}$ such that $\mu^{3}+\mu+1=\omega^{2}+\omega+1=0$. The algebra $\mathcal{A}_{2}$ is decomposed as the direct sum of six ideals, which are generated by the following idempotents:

$$
\begin{aligned}
& \mathcal{K}_{\{(1,1)\}}=\left\langle\left[\begin{array}{lllllll}
1 & 1 & 1 & 1 & 1 & 1 & 1 \\
1 & 1 & 1 & 1 & 1 & 1 & 1 \\
1 & 1 & 1 & 1 & 1 & 1 & 1
\end{array}\right]\right\rangle, \quad \mathcal{K}_{C_{2}((\mu, 1))}=\left\langle\left[\begin{array}{lllllll}
1 & 1 & 1 & 0 & 1 & 0 & 0 \\
1 & 1 & 1 & 0 & 1 & 0 & 0 \\
1 & 1 & 1 & 0 & 1 & 0 & 0
\end{array}\right]\right\rangle, \\
& \mathcal{K}_{C_{2}\left(\left(\mu^{6}, 1\right)\right)}=\left\langle\left[\begin{array}{lllllll}
1 & 0 & 0 & 1 & 0 & 1 & 1 \\
1 & 0 & 0 & 1 & 0 & 1 & 1 \\
1 & 0 & 0 & 1 & 0 & 1 & 1
\end{array}\right]\right\rangle, \mathcal{K}_{C_{2}((1, \omega))}=\left\langle\left[\begin{array}{lllllll}
0 & 0 & 0 & 0 & 0 & 0 & 0 \\
1 & 1 & 1 & 1 & 1 & 1 & 1 \\
1 & 1 & 1 & 1 & 1 & 1 & 1
\end{array}\right]\right\rangle \text {, }
\end{aligned}
$$

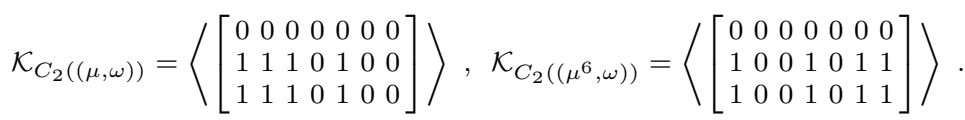

- If $q=4$, then the set of 4 -classes is the following one:

$$
\begin{aligned}
\mathcal{C}_{4}=\{ & \{(1,1)\},\left\{(\mu, 1),\left(\mu^{2}, 1\right),\left(\mu^{4}, 1\right)\right\},\left\{\left(\mu^{6}, 1\right),\left(\mu^{5}, 1\right),\left(\mu^{3}, 1\right)\right\}, \\
& \{(1, \omega)\},\left\{\left(1, \omega^{2}\right)\right\},\left\{(\mu, \omega),\left(\mu^{2}, \omega\right),\left(\mu^{4}, \omega\right)\right\} \\
& \left\{\left(\mu, \omega^{2}\right),\left(\mu^{2}, \omega^{2}\right),\left(\mu^{4}, \omega^{2}\right)\right\},\left\{\left(\mu^{6}, \omega\right),\left(\mu^{5}, \omega\right),\left(\mu^{3}, \omega\right)\right\} \\
& \left.\left\{\left(\mu^{6}, \omega^{2}\right),\left(\mu^{5}, \omega^{2}\right),\left(\mu^{3}, \omega^{2}\right)\right\}\right\}
\end{aligned}
$$

Observe that each 2-class of even size splits into two 4-classes. The decomposition of $\mathcal{A}_{4}$ is given by the following direct sum of 9 ideals generated by the idempotents

$$
\begin{aligned}
& \mathcal{I}_{\{(1,1)\}}=\left\langle\left[\begin{array}{lllllll}
1 & 1 & 1 & 1 & 1 & 1 & 1 \\
1 & 1 & 1 & 1 & 1 & 1 & 1 \\
1 & 1 & 1 & 1 & 1 & 1 & 1
\end{array}\right]\right\rangle, \quad \mathcal{I}_{C_{4}((\mu, 1))}=\left\langle\left[\begin{array}{lllllll}
1 & 1 & 1 & 0 & 1 & 0 & 0 \\
1 & 1 & 1 & 0 & 1 & 0 & 0 \\
1 & 1 & 1 & 0 & 1 & 0 & 0
\end{array}\right]\right\rangle,
\end{aligned}
$$

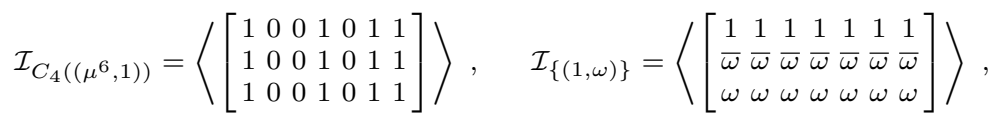

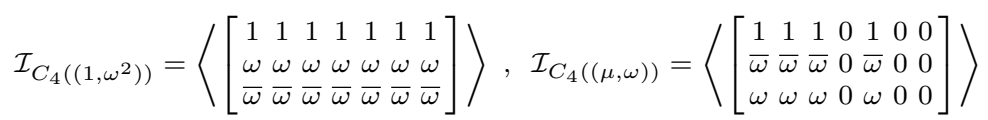

$$
\begin{aligned}
& \mathcal{I}_{C_{4}\left(\left(\mu, \omega^{2}\right)\right)}=\left\langle\left[\begin{array}{ccccccc}
1 & 1 & 1 & 0 & 1 & 0 & 0 \\
\omega & \omega & \omega & 0 & \omega & 0 & 0 \\
\bar{\omega} & \bar{\omega} & \bar{\omega} & 0 & \bar{\omega} & 0 & 0
\end{array}\right]\right\rangle, \quad \mathcal{I}_{C_{4}\left(\left(\mu^{6}, \omega\right)\right)}=\left\langle\left[\begin{array}{ccccccc}
1 & 0 & 0 & 1 & 0 & 1 & 1 \\
\bar{\omega} & 0 & 0 & \bar{\omega} & 0 & \bar{\omega} & \bar{\omega} \\
\omega & 0 & 0 & \omega & 0 & \omega & \omega
\end{array}\right]\right\rangle \text {, } \\
& \mathcal{I}_{C_{4}\left(\left(\mu^{6}, \omega^{2}\right)\right)}=\left\langle\left[\begin{array}{ccccccc}
1 & 0 & 0 & 1 & 0 & 1 & 1 \\
\omega & 0 & 0 & \omega & 0 & \omega & \omega \\
\bar{\omega} & 0 & 0 & \bar{\omega} & 0 & \bar{\omega} & \bar{\omega}
\end{array}\right]\right\rangle \text {. }
\end{aligned}
$$

(where $\bar{\omega}=\omega^{2}$ ). Notice that $\mathcal{A}_{2}$ is identified with the subalgebra $S$ of $\mathcal{A}_{4}$ of elements whose normal form has coefficients 0 or 1 . 
2.2 Relation between the decompositions of $\mathcal{A}_{4}$ and $\mathcal{A}_{2}$

From now on, let us fix $q=4$. In the following, we will describe the relation between the structures of the algebras $\mathcal{A}_{4}$ and $\mathcal{A}_{2}$. This will lead us, in the following section, to the description of the additive multivariable codes over $\mathbb{F}_{4}$. Since these decompositions are based on classes of roots, we first establish the relation between the 2 -classes $C_{2}(\mu)$ and the 4 -classes $C_{4}(\mu)$

Lemma 2 Let $\mu_{i}$ be a root of the polynomial $t_{i}\left(X_{i}\right), 1 \leq i \leq r$. Let $q_{i}=\operatorname{deg}\left(\operatorname{Irr}\left(\mu_{i}, \mathbb{F}_{4}\right)\right)$ and $k_{i}=\operatorname{deg}\left(\operatorname{Irr}\left(\mu_{i}, \mathbb{F}_{2}\right)\right)$. If $\mu=\left(\mu_{1}, \ldots, \mu_{r}\right)$, then:

1. If $k_{i}$ is odd for all $1 \leq i \leq r$, then $C_{2}(\mu)=C_{4}(\mu)$, and it has size l.c.m. $\left(k_{1}, \ldots, k_{r}\right)$.

2. If there exists $i \in\{1, \ldots, r\}$ such that $k_{i}$ is even, then $C_{2}(\mu)=C_{4}(\mu) \cup C_{4}\left(\mu^{2}\right)$, where $\mu^{2}=\left(\mu_{1}^{2}, \ldots, \mu_{r}^{2}\right)$. The union is disjoint and both classes have equal size l.c.m $\left(q_{1}, \ldots, q_{r}\right)$.

The subset of 2 -classes in the first case will be denoted $\mathcal{C}_{2}^{o}$, where as the 2 -classes in the second case will be denoted $\mathcal{C}_{2}^{e}$.

Proof If $k_{i}$ is odd for all $1 \leq i \leq r$, then the minimal polynomials $\left\{\operatorname{Irr}\left(\mu_{i}, \mathbb{F}_{2}\right)\right\}_{i=1}^{r}$ are also irreducible in $\mathbb{F}_{4}\left[X_{i}\right]$, and $q_{i}=k_{i}$. So, the result straightforwardly follows from Proposition 1.

In the second case assume w.l.o.g. that $k_{i}$ is even, for all $1 \leq i \leq k$, and odd for all $k+1 \leq i \leq r$ (with $k \geq 1$ ). Then, for all $1 \leq i \leq k$, the minimal polynomial $\operatorname{Irr}\left(\mu_{i}, \mathbb{F}_{2}\right)$ is the product of two irreducible polynomials of degree $q_{i}$ in $\mathbb{F}_{4}\left[X_{i}\right]$. The roots of these polynomials constitute the 4 -classes $C_{4}\left(\mu_{i}\right)$ and $C_{4}\left(\mu_{i}^{2}\right)$ respectively. Clearly, these are disjoint classes of equal size $q_{i}$ and $C_{2}\left(\mu_{i}\right)=C_{4}\left(\mu_{i}\right) \cup C_{4}\left(\mu_{i}^{2}\right)$. Therefore, $\mu^{2}$ does not belong to $C_{4}(\mu)$ and so $C_{4}\left(\mu^{2}\right)$ is disjoint with it. According to Proposition 1,

$$
\begin{aligned}
\left|C_{2}(\mu)\right|= & \operatorname{l} \cdot \operatorname{c} \cdot \mathrm{m}\left(k_{1}, \ldots, k_{r}\right)=1 \cdot \operatorname{c} \cdot \mathrm{m}\left(2 q_{1}, \ldots, 2 q_{k}, q_{k+1}, \ldots, q_{r}\right)= \\
& =2 \operatorname{l} \cdot \operatorname{c.m}\left(q_{1}, \ldots, q_{k}, q_{k+1}, \ldots, q_{r}\right)=2\left|C_{4}(\mu)\right|
\end{aligned}
$$

and also

$$
\left|C_{2}(\mu)\right|=\left|C_{2}\left(\mu^{2}\right)\right|=2\left|C_{4}\left(\mu^{2}\right)\right|
$$

hence we obtain the result.

Now, we can decompose the rings $\mathcal{A}_{4}$ and $\mathcal{A}_{2}$ into minimal ideals using Theorem 1. The decomposition for $\mathcal{A}_{4}$ is the following one:

$$
\mathcal{A}_{4} \cong \bigoplus_{C \in \mathcal{C}_{4}} \mathcal{I}_{C}
$$

where, for all $C=C_{4}(\mu) \in \mathcal{C}_{4}$, we denote the ideal $\mathcal{I}_{C}=\left\langle e_{C}+I\right\rangle$, which is isomorphic to the finite field $\mathbb{F}_{4}(\mu)$ of size $4^{|C|}$, and $e_{C}+I$ is idempotent.

On the other hand, the decomposition of the ring $\mathcal{A}_{2}$ into minimal ideals is

$$
\mathcal{A}_{2} \cong \bigoplus_{C \in \mathcal{C}_{2}} \mathcal{K}_{C}=\left(\bigoplus_{C \in \mathcal{C}_{2}^{o}} \mathcal{K}_{C}\right) \oplus\left(\bigoplus_{C \in \mathcal{C}_{2}^{e}} \mathcal{K}_{C}\right)
$$

where, for all $C=C_{2}(\mu) \in \mathcal{C}_{2}^{o} \cup \mathcal{C}_{2}^{e}, \mathcal{K}_{C}=\left\langle l_{C}+J\right\rangle$ is isomorphic to the field $\mathbb{F}_{2}(\mu)$, and so its size is $2^{|C|}$, and the element $l_{C}+J$ is idempotent. 
Next, let us consider the ring automorphism $\tau: \mathbb{F}_{4}\left[X_{1}, \ldots, X_{r}\right] \rightarrow \mathbb{F}_{4}\left[X_{1}, \ldots, X_{r}\right]$, where

$$
\tau\left(\sum_{i_{1}, \ldots, i_{r}} a_{i_{1} \ldots, i_{r}} X_{1}^{i_{1}} \cdots X_{r}^{i_{r}}\right)=\sum_{i_{1}, \ldots, i_{r}} a_{i_{1} \ldots, i_{r}}^{2} X_{1}^{i_{1}} \cdots X_{r}^{i_{r}}
$$

Since $t_{i}\left(X_{i}\right) \in \mathbb{F}_{2}\left[X_{i}\right]$, for all $1 \leq i \leq r$, we have that $\tau\left(t_{i}\left(X_{i}\right)\right)=t_{i}\left(X_{i}\right)$, and so $\operatorname{ker}\left(\pi_{4} \circ \tau\right) \subseteq \operatorname{ker} \pi_{4}$ (where $\pi_{4}: \mathbb{F}_{4}\left[X_{1}, \ldots, X_{r}\right] \rightarrow \mathcal{A}_{4}$ is the canonical epimorphism). Hence, there exists a ring homomorphism $\tilde{\tau}: \mathcal{A}_{4} \rightarrow \mathcal{A}_{4}$ such that $\tilde{\tau}(f+I)=\tau(f)+I$, for all $f+I \in \mathcal{A}_{4}$. This map is injective, since for all $f \notin I$, we have that $f+I=N F(f)+I$, where $0 \neq N F(f) \in \mathbb{F}_{4}\left[X_{1}, \ldots, X_{r}\right]$, and $\operatorname{deg}_{X_{i}}(N F(f))<n_{i}$, and so $\tilde{\tau}(f+I)=\tau(N F(f))+I \neq I$. Clearly, this fact implies that $\tilde{\tau}$ is also bijective and so it is a ring automorphism. Its fixed subring is $S$.

Proposition 4 Let $\mathcal{A}_{4} \cong \bigoplus_{C \in \mathcal{C}_{4}} \mathcal{I}_{C}$ and $\mathcal{A}_{2} \cong \bigoplus_{C \in \mathcal{C}_{2}^{\circ}} \mathcal{K}_{C} \oplus \bigoplus_{C \in \mathcal{C}_{2}^{e}} \mathcal{K}_{C}$ be the decompositions of $\mathcal{A}_{4}$ and $\mathcal{A}_{2}$ into minimal ideals, and let $S$ be the subring of $\mathcal{A}_{4}$, isomorphic to $\mathcal{A}_{2}$, fixed by $\tilde{\tau}$. If $\varphi$ is the embedding of $\mathcal{A}_{2}$ in $\mathcal{A}_{4}$ (see Proposition 3), then the following hold.

1. If $C \in \mathcal{C}_{2}^{o}$, then:

(a) $C \in \mathcal{C}_{4}, \tilde{\tau}\left(\mathcal{I}_{C}\right)=\mathcal{I}_{C}$, and $\left.\tilde{\tau}\right|_{\mathcal{I}_{C}}$ is a field automorphism of order 2.

(b) $\varphi\left(\mathcal{K}_{C}\right)=\mathcal{I}_{C} \cap S$, so it is the subfield of $\mathcal{I}_{C}$ isomorphic to $\mathbb{F}_{2^{|C|} \mid}$.

(c) $\mathcal{I}_{C}$ is 2 -dimensional $\mathcal{K}_{C}$-vector space.

2. If $C \in \mathcal{C}_{2}^{e}$, then:

(a) $C=C_{1} \cup C_{2}$, where $C_{1}, C_{2} \in \mathcal{C}_{4}, \tilde{\tau}\left(\mathcal{I}_{C_{1}}\right)=\mathcal{I}_{C_{2}}, \tilde{\tau}\left(\mathcal{I}_{C_{2}}\right)=\mathcal{I}_{C_{1}}$, and $\left.\tilde{\tau}\right|_{\mathcal{I}_{C_{1}} \rightarrow \mathcal{I}_{C_{2}}}$ is a field isomorphism.

(b) $\varphi\left(\mathcal{K}_{C}\right)=\left(\mathcal{I}_{C_{1}} \oplus \mathcal{I}_{C_{2}}\right) \cap S=\left\{(f+\tau(f))+I \mid f \in \mathcal{I}_{C_{1}}\right\}$ is isomorphic to the field $\mathcal{I}_{C_{1}} \cong \mathbb{F}_{4^{\left|C_{1}\right|}}=\mathbb{F}_{2^{|C|} \mid}$.

(c) $\mathcal{I}_{C_{1}} \oplus \mathcal{I}_{C_{2}}$ is 2 -dimensional $\mathcal{K}_{C^{-} \text {-vector space. }}$

Proof Notice that, if $\mu$ is a root of $f\left(X_{1}, \ldots, X_{r}\right)$, then $\mu^{2}$ is a root of the polynomial $\tau\left(f\left(X_{1}, \ldots, X_{r}\right)\right)$, and $\tau^{2}\left(f\left(X_{1}, \ldots, X_{r}\right)\right)=f\left(X_{1}, \ldots, X_{r}\right)$. So, $\tilde{\tau}$ induces a permutation of order 2 of the idempotents $\left\{e_{C}+I\right\}_{C \in \mathcal{C}_{4}}$, so that $\tilde{\tau}\left(e_{C}+I\right)=e_{C}+I$ if $C \in \mathcal{C}_{2}^{o}$, and $\tilde{\tau}\left(e_{C_{1}}+I\right)=e_{C_{2}}+I, \tilde{\tau}\left(e_{C_{2}}+I\right)=e_{C_{1}}+I$, if $C_{1} \cup C_{2}=C \in \mathcal{C}_{2}^{e}$, where $C_{1}, C_{2} \in \mathcal{C}_{4}$. This permutation is translated into the field isomorphisms of the statements 1.(a) and 2.(a).

For the statement 1.(b), it suffices to notice that $C=C_{4}(\mu)=C_{2}(\mu)$, so that $\varphi\left(l_{C}+J\right)=e_{C}+I$, and $\varphi\left(\mathcal{K}_{C}\right) \subseteq \mathcal{I}_{C} \cap S$. A counting argument let us conclude the desired equality.

Let us now show the statement 2.(b). The zeros of the idempotent $\varphi\left(l_{C}+J\right)$ are exactly $H_{1} \times \ldots \times H_{r} \backslash C$. The zeros of $e_{C_{i}}+I(i=1,2)$ are $H_{1} \times \ldots \times H_{r} \backslash C_{i}$, and so the idempotent $e_{C_{1}}+e_{C_{2}}+I \in \mathcal{I}_{C_{1}} \oplus \mathcal{I}_{C_{2}}$ has zeros $H_{1} \times \ldots \times H_{r} \backslash\left(C_{1} \cup C_{2}\right)=$ $H_{1} \times \ldots \times H_{r} \backslash C$. Hence $\varphi\left(l_{C}+J\right) \subseteq\left(\mathcal{I}_{C_{1}} \oplus \mathcal{I}_{C_{2}}\right) \cap S$, and the equality follows from another counting argument.

Finally, the statements $(c)$ follow from the fact that $\varphi$ is an embedding of the finite field $\mathcal{K}_{C}$ (of size $2^{|C|}$ ) into the ring $\mathcal{I}_{C}\left(\right.$ if $C \in \mathcal{C}_{2}^{o}$ ), or $\mathcal{I}_{C_{1}} \oplus \mathcal{I}_{C_{2}}$ (if $C \in \mathcal{C}_{2}^{e}$ ), and so they can be regarded as $\mathcal{K}_{C}$-vector spaces. Since both have size $4^{|C|}$, we conclude that their dimension is 2 . 
Remark 5 For $C \in \mathcal{C}_{2}^{o}$, the idempotent $e_{C}+I$ is the identity of $\varphi\left(\mathcal{K}_{C}\right)$, and so $e_{C}+I \in \varphi\left(\mathcal{K}_{C}\right)=\left\langle X_{1}^{i_{1}} \ldots X_{r}^{i_{r}} e_{C}+I \mid 0 \leq i_{l} \leq \operatorname{deg}\left(t_{l}\left(X_{l}\right)\right)\right\rangle_{\mathbb{F}_{2}}$. On the other hand, for $C_{1} \cup C_{2}=C \in \mathcal{C}_{2}^{e}$ the idempotent $e_{C_{1}}+e_{C_{2}}+I$ is the identity of $\varphi\left(\mathcal{K}_{C}\right)$, and so $\varphi\left(\mathcal{K}_{C}\right)=\left\langle X_{1}^{i_{1}} \ldots X_{r}^{i_{r}}\left(e_{C_{1}}+e_{C_{2}}\right)+I \mid 0 \leq i_{l} \leq \operatorname{deg}\left(t_{l}\left(X_{l}\right)\right)\right\rangle_{\mathbb{F}_{2}}$.

Example 2 (Example 1 cont'd) Here is the list of odd and even 2-classes:

$$
\begin{aligned}
\mathcal{C}_{2}^{o}= & \left.\left\{\{(1,1)\},\left\{(\mu, 1),\left(\mu^{2}, 1\right),\left(\mu^{4}, 1\right)\right\},\left\{\left(\mu^{6}, 1\right),\left(\mu^{5}, 1\right),\left(\mu^{3}, 1\right)\right\}\right\}\right\} \\
C_{2}^{e}=\left\{(1, \omega),\left(1, \omega^{2}\right)\right\},\left\{(\mu, \omega),\left(\mu^{2}, \omega\right),\left(\mu^{4}, \omega\right),\left(\mu, \omega^{2}\right),\left(\mu^{2}, \omega^{2}\right),\left(\mu^{4}, \omega^{2}\right)\right\}, & \left.\left\{\left(\mu^{6}, \omega\right),\left(\mu^{5}, \omega\right),\left(\mu^{3}, \omega\right),\left(\mu^{6}, \omega^{2}\right),\left(\mu^{5}, \omega^{2}\right),\left(\mu^{3}, \omega^{2}\right)\right\}\right\}
\end{aligned}
$$

The ideal

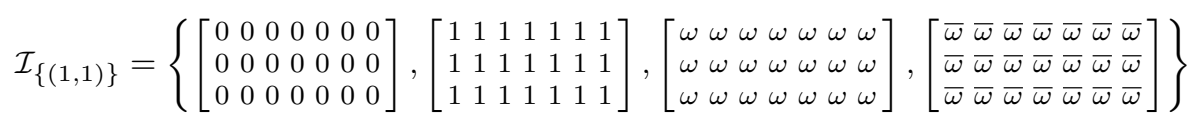

(where $\bar{w}=w^{2}$ ) is isomorphic to $\mathbb{F}_{4}$, and it is a 2-dimensional vector space over

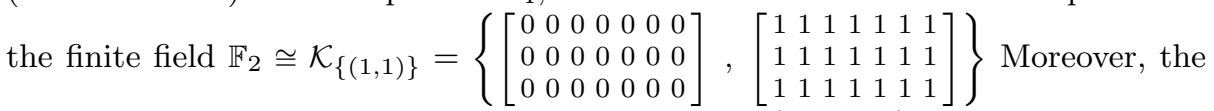
elements in $\mathcal{I}_{\{(1,1)\}}$ with coefficients equal to 0 or 1 (i.e, in $S$ ) are exactly those of $\mathcal{K}_{\{(1,1)\}}$.

The ideal $\mathcal{I}_{C_{4}((\mu, 1))}$ is isomorphic to $\mathbb{F}_{4^{3}}$, and it is a 2 -dimensional vector space over the finite field

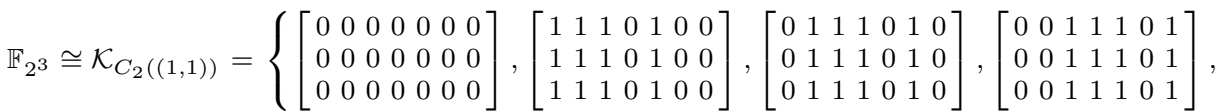

$$
\begin{aligned}
& \left.\left[\begin{array}{lllllll}
1 & 0 & 0 & 1 & 1 & 1 & 0 \\
1 & 0 & 0 & 1 & 1 & 1 & 0 \\
1 & 0 & 0 & 1 & 1 & 1 & 0
\end{array}\right],\left[\begin{array}{lllllll}
0 & 1 & 0 & 0 & 1 & 1 & 1 \\
0 & 1 & 0 & 0 & 1 & 1 & 1 \\
0 & 1 & 0 & 0 & 1 & 1 & 1
\end{array}\right],\left[\begin{array}{lllllll}
1 & 0 & 1 & 0 & 0 & 1 & 1 \\
1 & 0 & 1 & 0 & 0 & 1 & 1 \\
1 & 0 & 1 & 0 & 0 & 1 & 1
\end{array}\right],\left[\begin{array}{lllllll}
1 & 1 & 0 & 1 & 0 & 0 & 1 \\
1 & 1 & 0 & 1 & 0 & 0 & 1 \\
1 & 1 & 0 & 1 & 0 & 0 & 1
\end{array}\right]\right\}
\end{aligned}
$$

The ideals related to the 2 and 4 -classes of $\left(\mu^{6}, 1\right)$ behave similarly.

On the other hand, all the even classes follow the pattern of the 2-class $\left\{(1, \omega),\left(1, \omega^{2}\right)\right\}$. Namely, the ideal

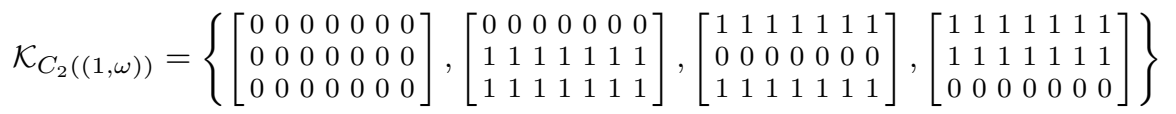

is isomorphic to $\mathbb{F}_{4}$, and the direct sum of the ideals

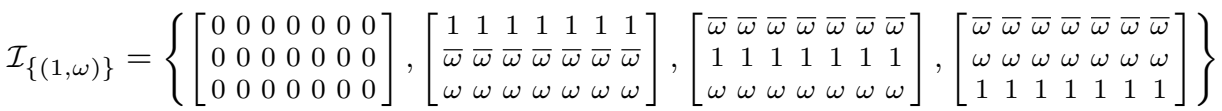

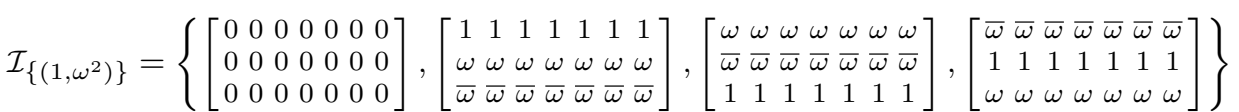

is a vector space over it. 


\section{Additive multivariable codes}

Next we take advantage of the decomposition of the rings $\mathcal{A}_{4}, \mathcal{A}_{2}$, and their relation in order to obtain a complete description of an additive semisimple multivariable code $\mathcal{D} \subseteq \mathcal{A}_{4}$. From now on we assume that $\mathcal{A}_{4} \cong \bigoplus_{C \in \mathcal{C}_{4}} \mathcal{I}_{C}$ and $\mathcal{A}_{2} \cong \bigoplus_{C \in \mathcal{C}_{2}^{o}} \mathcal{K}_{C} \oplus \bigoplus_{C \in \mathcal{C}_{2}^{e}} \mathcal{K}_{C}$ are the decompositions of $\mathcal{A}_{4}$ and $\mathcal{A}_{2}$ into minimal ideals, that $\varphi$ is the embedding of $\mathcal{A}_{2}$ in $\mathcal{A}_{4}$, and that $S=\varphi\left(\mathcal{A}_{2}\right)$ is the subring of $\mathcal{A}_{4}$ fixed by the ring automorphism $\tilde{\tau}$. The main result in this section is the following.

Theorem 2 Let $\mathcal{D} \subseteq \mathcal{A}_{4}$ be an additive semisimple code, i.e., a $S$-submodule of $\mathcal{A}_{4}$.

1. If $C \in C_{2}^{o}$, then the set $\mathcal{D}_{C}=\mathcal{D} \cap \mathcal{I}_{C}$ is a $\mathcal{K}_{C}$-vector subspace of $\mathcal{I}_{C}$ of dimension $0 \leq s_{C} \leq 2$.

2. If $C \in C_{2}^{e}$, with $C=C_{1} \cup C_{2}$, and $C_{1}, C_{2} \in \mathcal{C}_{4}$, then the set $\mathcal{D}_{C}=\mathcal{D} \cap\left(\mathcal{I}_{C_{1}} \oplus \mathcal{I}_{C_{2}}\right)$ is a $\mathcal{K}_{C}$-vector subspace of dimension $0 \leq s_{C} \leq 2$.

3. $\mathcal{D}=\bigoplus_{C \in \mathcal{C}_{2}} \mathcal{D}_{C},|\mathcal{D}|=\prod_{C \in \mathcal{C}_{2}} 2^{s_{C}}$, and the decomposition is unique.

Proof $1 . \mathcal{D}_{C}$ is an additive subgroup of the ideal $\mathcal{I}_{C}$, since $\mathcal{D}$ is a code. Moreover, because it is an additive code, it is invariant under multiplication by elements in $S$ so, in particular, by elements in $\mathcal{I}_{C} \cap S=\varphi\left(\mathcal{K}_{C}\right)$. Henceforth, since $\varphi$ is injective, $\mathcal{D}_{C}$ can be viewed as a vector space over the finite field $\mathcal{K}_{C}$, i.e., as a vector subspace of $\mathcal{I}_{C}$. Because $\operatorname{dim}_{\mathcal{K}_{C}} \mathcal{I}_{C}=2$, we conclude the condition on the dimension of $\mathcal{D}_{C}$.

2. The argument above applies also in this case replacing $\mathcal{I}_{C}$ by $\mathcal{I}_{C_{1}} \oplus \mathcal{I}_{C_{2}}$.

3. For all $C \in \mathcal{C}_{2}$ we have that $\mathcal{D}_{C} \subseteq \mathcal{D}$, and so $\bigoplus_{C \in \mathcal{C}_{2}} \mathcal{D}_{C} \subseteq \mathcal{D}$. Conversely, if $m+I \in \mathcal{D}$, then

$$
\begin{aligned}
& m+I=(1+I)(m+I)=\left(\bigoplus_{C \in \mathcal{C}_{4}}\left(e_{C}+I\right)\right)(m+I)= \\
= & \left(\bigoplus_{C \in \mathcal{C}_{2}^{o}}\left(e_{C}+I\right) \oplus \bigoplus_{\substack{C_{1}, C_{2} \in \mathcal{C}_{4} \\
C_{1} \cup C_{2} \in \mathcal{C}_{2}^{e}}}\left(e_{C_{1}}+e_{C_{2}}+I\right)\right)(m+I) \\
= & \bigoplus_{C \in \mathcal{C}_{2}^{o}}\left(e_{C} m+I\right) \oplus \bigoplus_{\substack{C_{1}, C_{2} \in \mathcal{C}_{4} \\
C_{1} \cup C_{2} \in \mathcal{C}_{2}^{e}}}\left(\left(e_{C_{1}}+e_{C_{2}}\right) m+I\right) \in \bigoplus_{C \in \mathcal{C}_{2}} \mathcal{D}_{C}
\end{aligned}
$$

since $e_{C} m+I \in \mathcal{D}_{C}$ (for all $C \in \mathcal{C}_{2}^{o}$ ), and $\left(e_{C_{1}}+e_{C_{2}}\right) m+I \in \mathcal{D}_{C}$ (for all $\left.C_{1} \cup C_{2}=C \in \mathcal{C}_{2}^{e}\right)$. Clearly, $|D|=\prod_{C \in \mathcal{C}_{2}} 2^{s_{C}}$.

Finally, let us show that this decomposition is unique. Let $\mathcal{D}=\bigoplus_{C \in \mathcal{C}_{2}} \mathcal{E}_{C}$ where $\mathcal{E}_{C} \subseteq \mathcal{I}_{C}$, if $C \in \mathcal{C}_{2}^{o}$, and $\mathcal{E}_{C} \subseteq \mathcal{I}_{C_{1}} \oplus I_{C_{2}}$, if $C_{1} \cup C_{2}=C \in \mathcal{C}_{2}^{e}$. Then, $\mathcal{E}_{C} \subseteq \mathcal{I}_{C} \cap \mathcal{D}=\mathcal{D}_{C}$, in the first case, and $\mathcal{E}_{C} \subseteq\left(\mathcal{I}_{C_{1}} \oplus \mathcal{I}_{C_{2}}\right) \cap \mathcal{D}=\mathcal{D}_{C}$, in the second one. A counting argument on the sizes of these sets proves the result.

Corollary 1 The number of different additive semisimple codes in $\mathcal{A}_{4}$ is

$$
\prod_{C \in \mathcal{C}_{2}}\left(2^{|C|}+3\right)
$$

Of these, only $\prod_{C \in \mathcal{C}_{2}}\left(2^{|C|}+2\right)$ codes can be generated by a single codeword. 
Proof The number of subspaces in a 2-dimensional vector space over a finite field $\mathbb{F}_{q}$ is $1+\frac{q^{2}-1}{q-1}+1=q+3$. Hence, the result follows directly from the decomposition of the previous theorem.

A code $\mathcal{D}_{C}$ can be generated by a single codeword if and only if it is a cyclic $S$-submodule of $\mathcal{A}_{4}$. So, all the submodules $\mathcal{D}_{C}$ have to be also cyclic $S$-submodules, and the dimension of the vector subspace $\mathcal{D}_{C}$ over $\mathcal{K}_{C}$ has to be either 0 or 1 . The number of possible codes obtained from these subspaces is exactly $\prod_{C \in \mathcal{C}_{2}}\left(1+\left(2^{|C|}+1\right)\right)$.

\subsection{Hamming distance}

Next, we study the minimum distance of our codes. Let us first introduce the standard definitions.

Definition 6 If $f \in \mathbb{F}_{q}\left[X_{1}, \ldots, X_{r}\right]$, and $N F(f)=\sum_{i=1}^{r} f_{\alpha_{1} \ldots \alpha_{r}} X_{1}^{\alpha_{1}} \cdots X_{r}^{\alpha_{r}}$ is its normal form w.r.t. $I$, then we define the Hamming weight of $f+I$ (denoted by $\operatorname{wt}(f+I))$, as the cardinality of $\operatorname{supp}(N F(f))=\left\{\left(\alpha_{1}, \ldots, \alpha_{r}\right) \mid f_{\alpha_{1} \ldots \alpha_{r}} \neq 0\right\}$, the support of $N F(f)$.

The minimum distance of an additive (semisimple) multivariable code $\mathcal{D} \subseteq \mathcal{A}_{4}$ is defined as the minimum Hamming weight of the nonzero elements in $\mathcal{D}$, and it is denoted by $d(\mathcal{D})$.

The study of the codes with the best Hamming distance for given parameters is one of the central problems in Coding Theory. A good source of bounds and examples of the best known codes (block linear, and quantum error correcting codes) can be found in Code Tables [6]. There exist several bounds on distances for classical multivariable semisimple codes over fields (BCH, Hartmann-Tzeng, Roos, ...) [13]. These bounds can be stated in the additive case due to the following fact:

Proposition 5 Let $\mathcal{D} \subseteq \mathcal{A}_{4}$ be an additive abelian code, and let $T=\cup_{i=1}^{l} C_{i}$ be a union of $C_{4}$-classes $C_{i}=C_{4}\left(\mu_{i}\right)$ such that: $C_{i} \in T$ if and only if

$-C_{i} \in C_{2}^{o}$ and $\mathcal{D}_{C} \neq 0$

or

$-C_{i} \subseteq C^{\prime} \in C_{2}^{e}$ and $\mathcal{D}_{C^{\prime}} \neq 0$

Then $d(\mathcal{D}) \geq d\left(\mathcal{D}^{*}\right)$, where $\mathcal{D}^{*}$ is the classical multivariable code in $\mathcal{A}_{4}$ with set of defining roots equal to $T$ [13].

Proof It is enough to notice that, since $T$ is the set of defining roots of $\mathcal{D}^{*}$, then $\mathcal{D}_{C_{i}}=\mathcal{I}_{C_{i}}$, for all $i=1, \ldots, l$. Therefore $\mathcal{D} \subseteq D^{*}$ and the conclusion follows.

Remark 6 1. In view of this result, if we used the classical approach for multivariable codes, the computation of the minimum distance of a multivariable additive abelian code in $r$ variables would be reduced to computations of minimum distances of classical multivariable semisimple codes over a finite field in less number of variables ([13][Proposition 8, Chapter 6] )

2. There might exist additive codes with a greater Hamming distance that the one stated by the former bound, as the following example shows. Generally, these codes are not multivariable codes in the classical sense. 
Example 3 (Example 1 cont'd) We apply Theorem 2 to construct an additive code by choosing suitable chunks (i.e., subcodes) in the components of the decomposition of $\mathcal{A}_{4}$, in the following way:

- In the component $\mathcal{I}_{\{(1,1)\}}$, we choose

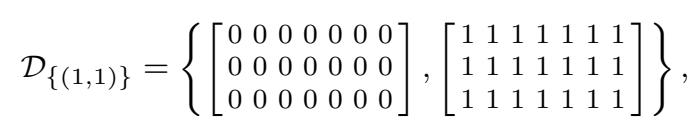

which is a 1 -dimensional vector space over $\mathcal{K}_{\{(1,1)\}}$.

- In the ideals $\mathcal{I}_{C_{4}((\mu, 1))}$ and $\mathcal{I}_{C_{4}\left(\left(\mu^{6}, 1\right)\right)}$, we choose 1 -dimensional vector spaces over $\mathcal{K}_{C_{2}((\mu, 1))}$ and $\mathcal{K}_{C_{2}((\mu, 1))}$, respectively. According to the proof of Corollary 1, we have $9\left(=\frac{8^{2}-1}{8-1}\right)$ choices for each of them. Let us take

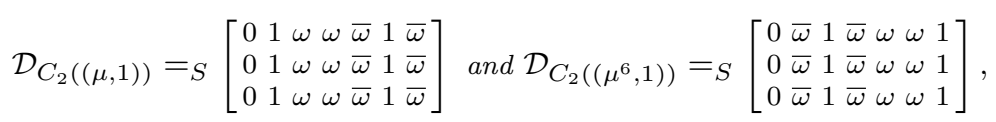

respectively.

Let us have a more detailed look at the subcode $D_{C_{2}((\mu, 1)) \text {. Since it is only contained }}$ in the component $\mathcal{I}_{C_{4}((\mu, 1))}$, which is generated by the polynomial $\left(1+X_{1}+X_{1}^{2}+\right.$ $\left.X_{1}^{4}\right)\left(1+X_{2}+X_{2}^{2}\right)$, Proposition 5 ensures a minimum distance of 12 , according to the BCH bound. But Sage computations [16] show that the actual distance is $d\left(\mathcal{D}_{C_{2}((\mu, 1))}\right)=18$.

- We simply choose $\mathcal{D}_{C_{2}((1, \omega))}=\left\{\left[\begin{array}{lllllll}0 & 0 & 0 & 0 & 0 & 0 & 0 \\ 0 & 0 & 0 & 0 & 0 & 0 & 0 \\ 0 & 0 & 0 & 0 & 0 & 0 & 0\end{array}\right]\right\}$.

- Finally, in the sums $\mathcal{I}_{C_{4}((\mu, \omega))} \oplus \mathcal{I}_{C_{4}\left(\left(\mu, \omega^{2}\right)\right)}$ and $\mathcal{I}_{C_{4}\left(\left(\mu^{6}, \omega\right)\right)} \oplus \mathcal{I}_{C_{4}\left(\left(\mu^{6}, \omega^{2}\right)\right)}$, we also consider 1 -dimensional vector spaces over $\mathcal{K}_{C_{2}((\mu, \omega))}$ and $\mathcal{K}_{C_{2}\left(\left(\mu^{6}, \omega\right)\right) \text {, re- }}$ spectively. Corollary 1 shows that a total amount of 65 possibilities are allowed for each component. Let us choose

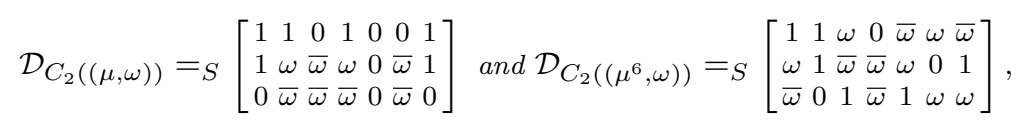

respectively.

The additive code

$$
\mathcal{D}=\mathcal{D}_{\{(1,1)\}} \oplus \mathcal{D}_{C_{2}((\mu, 1))} \oplus \mathcal{D}_{C_{2}\left(\left(\mu^{6}, 1\right)\right)} \oplus \mathcal{D}_{C_{2}((1, \omega))} \oplus \mathcal{D}_{C_{2}\left(\left(\mu^{6}, \omega\right)\right)} \oplus D_{C_{2}\left(\left(\mu^{6}, \omega\right)\right)}
$$

of length 21 has $2^{19}=2^{1+3+3+0+6+6}$ codewords. Sage computations show that this code has distance $d(\mathcal{D})=7$.

\section{Duality for abelian codes}

In this section we describe the dual code of an additive abelian code $\mathcal{D}$. Following Lemma 1 , the elements of $\mathcal{A}_{4}$ can be uniquely represented as $f+I$, where $f=N F(f)=$ $\sum_{\alpha} f_{\alpha} X^{\alpha}, \alpha=\left(\alpha_{1}, \ldots, \alpha_{r}\right)$, and $X^{\alpha}=X_{1}^{\alpha_{1}} \ldots X_{r}^{\alpha_{r}}$ (notice that $0 \leq \alpha_{i}<n_{i}$, for all $1 \leq i \leq r)$. Let us introduce the function ${ }^{-}: \mathcal{A}_{4} \rightarrow \mathcal{A}_{4}$ that maps an element $f+I$ to $\overline{f+I}=\sum_{\alpha} f_{\alpha} X^{n-\alpha}+I$, where $n=\left(n_{1}, \ldots, n_{r}\right)$. It is a ring automorphism or order 
2. With this notation, the symmetric function used to define orthogonality is the trace inner product $\langle\cdot, \cdot\rangle: \mathcal{A}_{4} \times \mathcal{A}_{4} \rightarrow \mathbb{F}_{2}$ given by

$$
\langle f+I, g+I\rangle=\sum_{\alpha} \operatorname{Tr}\left(f_{\alpha} g_{\alpha}^{2}\right)=\sum_{\alpha}\left(f_{\alpha} g_{\alpha}^{2}+f_{\alpha}^{2} g_{\alpha}\right)
$$

where $\operatorname{Tr}: \mathbb{F}_{4} \rightarrow \mathbb{F}_{2}$ is the trace function. So, given an additive abelian code $\mathcal{D}$, its dual code is defined as $\mathcal{D}^{\perp}=\left\{f+I \in \mathcal{A}_{4} \mid\langle f+I, g+I\rangle=0\right.$ for all $\left.g+I \in \mathcal{D}\right\}$. A code $\mathcal{D}$ is self-orthogonal if $\mathcal{D} \subseteq \mathcal{D}^{\perp}$ and self-dual if $\mathcal{D}=\mathcal{D}^{\perp}$. In the next section we will characterize this kind of codes. by

For the study of duality it is useful to introduce the map $(\cdot, \cdot): \mathcal{A}_{4} \times \mathcal{A}_{4} \rightarrow S$ given

$$
(f+I, g+I)=(f+I) \overline{\tilde{\tau}(g+I)}+\tilde{\tau}(f+I) \overline{g+I},
$$

Notice that $(\cdot, \cdot)$ is well-defined, since $\tilde{\tau}(f+I) \overline{g+I}=\tilde{\tau}((f+I) \overline{\tilde{\tau}(g+I)})$, and so $(f+I, g+I)$ is contained in the subring of $\mathcal{A}_{4}$ fixed by $\tilde{\tau}$, i.e., in $S$. Moreover, $I=$ $0+I=(f+I, g+I)$ if and only if $(f+I) \overline{\tilde{\tau}(g+I)}=\tilde{\tau}(f+I) \overline{g+I}$ if and only if $(f+I) \overline{\tilde{\tau}(g+I)}$ is in $S$.

Notice that (1) resembles an Hermitian form, in the sense that it is $S$-linear in the first argument and $(g+I, f+I)=\overline{(f+I, g+I)}$, for all $f+I, g+I \in \mathcal{A}_{4}$. This map is related to the trace inner product by the following way:

Lemma $3(f+I, g+I)=\left(\sum_{\alpha}\left\langle f+I, X^{\alpha} g+I\right\rangle X^{\alpha}\right)+I$, for all $f+I, g+I \in \mathcal{A}_{4}$.

Proof

$$
\begin{gathered}
(f+I, g+I)=(f+I) \overline{\tilde{\tau}(g+I)}+\tilde{\tau}(f+I) \overline{g+I} \\
\left(\sum_{\beta} f_{\beta} X^{\beta}+I\right)\left(\sum_{\gamma} g_{\gamma}^{2} X^{n-\gamma}+I\right)+\left(\sum_{\beta} f_{\beta}^{2} X^{\beta}+I\right)\left(\sum_{\gamma} g_{\gamma} X^{n-\gamma}+I\right) \\
=\sum_{\beta} \sum_{\gamma}\left(f_{\beta} g_{\gamma}^{2}+f_{\beta}^{2} g_{\gamma}\right) X^{\beta+n-\gamma}+I=\sum_{\alpha}\left(\sum_{\gamma}\left(f_{\alpha+\gamma} g_{\gamma}^{2}+f_{\alpha+\gamma}^{2} g_{\gamma}\right)\right) X^{\alpha}+I \\
=\sum_{\alpha}\left(\sum_{\delta}\left(f_{\delta} g_{\delta-\alpha}^{2}+f_{\delta}^{2} g_{\delta-\alpha}\right)\right) X^{\alpha}+I=\left(\sum_{\alpha}\left\langle f+I, X^{\alpha} g+I\right\rangle X^{\alpha}\right)+I
\end{gathered}
$$

(we have applied the changes of index $\{\beta+n-\gamma=\alpha, \delta=\alpha+\gamma\}$, and we have taken the subscripts modulo $n$ )

So, $(f+I, g+I)=I$ if and only if $\langle r+I, g+I\rangle=0$ for all $r+I$ in ${ }_{S}(f+I)$, the $S$-submodule of $\mathcal{A}_{4}$ generated by $f+I$, i.e., if and only if $g+I$ is an element in the dual code of $S(f+I)$, the code spanned by $f+I$.

Notice that the composition of the ring automorphisms $\tilde{\tau}$ and - induces a permutation on the set $\mathcal{C}_{4}$, because if $\mu=\left(\mu_{1}, \ldots, \mu_{r}\right)$ is a root of $f+I$, then $\mu^{\prime}=$ $\left(\mu_{1}^{-2}, \ldots, \mu_{r}^{-2}\right)$ is a root of $\overline{\tilde{\tau}(f+I)}$. If $C=C_{4}(\mu)$ is the 4-class of the root $\mu$, then let us denote by $C^{\prime}=C_{4}\left(\mu^{\prime}\right)$ the 4 -class of $\mu^{\prime}$. Observe that, if we choose $C_{1}, C_{2} \in \mathcal{C}_{4}$ such that $C_{1} \cup C_{2} \in \mathcal{C}_{2}^{e}$, then $C_{1}^{\prime} \cup C_{2}^{\prime} \in \mathcal{C}_{2}^{e}$ too. So, if $C=C_{1} \cup C_{2} \in \mathcal{C}_{2}$, we also write $C^{\prime}=C_{1}^{\prime} \cup C_{2}^{\prime}$, and the permutation can be extended to 2 -classes. It is clear that $C_{2}\left(\left(1^{-2}, \ldots, 1^{-2}\right)\right)=C_{2}((1, \ldots, 1))$, and so $C_{2}((1, \ldots, 1))$ is a fixed point of this permutation. On the other hand, no other 2 -class in $\mathcal{C}_{2}^{o}$ is fixed. Namely, 
if $C_{2}((1, \ldots, 1)) \neq C_{2}(\mu)=C_{2}\left(\mu^{\prime}\right) \in \mathcal{C}_{2}^{o}$ then, since $C_{2}\left(\mu^{-1}\right)=C_{2}\left(\mu^{\prime}\right)$, we have $\mu^{-1} \in C_{2}(\mu)$. For all $\delta \neq(1, \ldots, 1)$, we have $\delta \neq \delta^{-1}$, and so $C_{2}(\mu)$ can be partitioned in pairs $\left(\delta, \delta^{-1}\right)$. Hence $C_{2}(\mu)$ must have even size, which is not possible.

Given $C \in \mathcal{C}_{2}^{o}$, and an element $f+I \in \mathcal{I}_{C}$ (or $C \in \mathcal{C}_{2}^{e}$, and an element $f+I \in$ $\mathcal{I}_{C_{1}} \oplus \mathcal{I}_{C_{2}}$ ), we define

$$
\mathcal{O}(f+I)=\left\{g+I \in \mathcal{I}_{C^{\prime}}\left(\text { alt. } g+I \in \mathcal{I}_{C_{1}^{\prime}} \oplus \mathcal{I}_{C_{2}^{\prime}}\right) \mid(f+I) \overline{\tilde{\tau}(g+I)} \in S\right\}
$$

We have the following result that completely describes the dual code $\mathcal{D}^{\perp}$ of a given abelian code $\mathcal{D}$.

Theorem 3 Let $\mathcal{D}$ be an abelian additive code. If $\mathcal{D}=\bigoplus_{C \in \mathcal{C}_{2}} \mathcal{D}_{C}$ is the unique decomposition of Theorem 2 , and $\mathcal{D}^{\perp}=\bigoplus_{C \in \mathcal{C}_{2}} \mathcal{D}_{C}^{\perp}$ is the unique decomposition of its dual code, then:

1. if $\mathcal{D}_{C}=0$, then $\mathcal{D}_{C^{\prime}}^{\perp}=\mathcal{I}_{C^{\prime}}\left(\right.$ if $\left.C \in \mathcal{C}_{2}^{o}\right)$, or $\mathcal{D}_{C^{\prime}}^{\perp}=\mathcal{I}_{C_{1}^{\prime}} \oplus \mathcal{I}_{C_{2}^{\prime}}\left(\right.$ if $\left.C=C_{1} \cup C_{2} \in \mathcal{C}_{2}^{e}\right)$;

2. $\mathcal{D}_{C^{\prime}}^{\perp}=0$ if $\mathcal{D}_{C}=\mathcal{I}_{C}$ (when $C \in \mathcal{C}_{2}^{o}$ ), or if $\mathcal{D}_{C}=\mathcal{I}_{C_{1}} \oplus \mathcal{I}_{C_{2}}$ (when $C=C_{1} \cup C_{2} \in$ $\left.\mathcal{C}_{2}^{e}\right)$

3. if $\mathcal{D}_{C}={ }_{S}(f+I)$ with $I \neq f+I$, then $\mathcal{D}_{C^{\prime}}^{\perp}=\mathcal{O}(f+I)$. In such a case:

(a) If $C \in \mathcal{C}_{2}^{o}$, then $\mathcal{O}(f+I)={ }_{S}(\overline{\tilde{\tau}(g+I)})$, where $g+I \in \mathcal{I}_{C}$ is the multiplicative inverse of $f+I$ in the field $\mathcal{I}_{C}$.

(b) If $C=C_{1} \cup C_{2} \in \mathcal{C}_{2}^{e}$, with $C_{1}, C_{2} \in \mathcal{C}_{4}$, then let us write $f+I=f_{1}+f_{2}+I$, where $f_{i}+I \in \mathcal{I}_{C_{i}}(i=1,2)$. If $f_{i}+I \neq I(i=1,2)$, then $\mathcal{O}(f+I)=_{S}$ $\left(\overline{\tilde{\tau}\left(g_{1}+g_{2}+I\right)}\right)$, where $g_{i}+I \in \mathcal{I}_{C_{i}}$ is the multiplicative inverse of $f_{i}+I$ in the field $\mathcal{I}_{C_{i}}(i=1,2)$. Otherwise, if $f_{i}+I \neq I$ (and $f_{3-i}+I=I$ ), then $\mathcal{O}(f+I)={ }_{S}\left(e_{C_{3-i}^{\prime}}+I\right)$, if $C \neq C^{\prime}$ or $\overline{\mathcal{I}_{C_{1}}} \neq \mathcal{I}_{C_{1}}$, or $\mathcal{O}(f+I)={ }_{S}\left(e_{C_{i}^{\prime}}+I\right)$, if $C=C^{\prime}$ and $\overline{\mathcal{I}_{C_{1}}}=\mathcal{I}_{C_{1}}$.

Summarizing, the possibilities given in Table 1 hold.

\begin{tabular}{|c|c|c|}
\hline & $\mathcal{D}_{C}$ & $\mathcal{D}_{C^{\prime}}^{\perp}$ \\
\hline \multirow{3}{*}{$C \in \mathcal{C}_{2}^{o}$} & $\{0\}$ & $\mathcal{I}_{C^{\prime}}$ \\
\hline & $S(f+I)$ & ${ }_{S}(\overline{\tilde{\tau}(g+I))}$ \\
\hline & $\mathcal{I}_{C}$ & $\{0\}$ \\
\hline \multirow{5}{*}{$C \in \mathcal{C}_{2}^{e}$} & $\{0\}$ & $\mathcal{I}_{C_{1}^{\prime}} \oplus \mathcal{I}_{C_{2}^{\prime}}$ \\
\hline & \multirow{2}{*}{$S\left(f_{i}+I\right)$} & ${ }_{S}\left(e_{C_{3-i}^{\prime}}^{\prime}+I\right)$, if $C \neq C^{\prime}$ or if $\overline{\mathcal{I}_{C_{1}}} \neq \mathcal{I}_{C_{1}}$ \\
\hline & & ${ }_{S}\left(e_{C_{i}^{\prime}}+I\right)$, if $C=C^{\prime}$ and $\overline{\mathcal{I}_{C_{1}}}=\mathcal{I}_{C_{1}}$ \\
\hline & $S_{S}\left(f_{1}+f_{2}+I\right)$ & ${ }_{S}\left(\overline{\tilde{\tilde{\tau}}\left(g_{1}+g_{2}+I\right)}\right.$ \\
\hline & $\mathcal{I}_{C_{1}} \oplus \mathcal{I}_{C_{2}}$ & $\{0\}$ \\
\hline
\end{tabular}

Table 1 Relation between the summands of orthogonal codes

Proof Observe that if $f+I \in \mathcal{I}_{C}$ (alt. $f+I \in \mathcal{I}_{C_{1}} \oplus \mathcal{I}_{C_{2}}$ ) and $h+I \notin \mathcal{I}_{C^{\prime}}$ (alt. $h+I \notin$ $\mathcal{I}_{C_{1}^{\prime}} \oplus \mathcal{I}_{C_{2}^{\prime}}$, then $\overline{\tilde{\tau}(h+I)} \in \mathcal{I}_{C^{*}} \neq \mathcal{I}_{C}\left(\right.$ alt. $\overline{\tilde{\tau}(h+I)} \in \mathcal{I}_{C_{1} *} \oplus \mathcal{I}_{C_{2} *} \neq \mathcal{I}_{C_{1}} \oplus \mathcal{I}_{C_{2}}$ ), and 
so $(f+I) \overline{\tilde{\tau}(h+I)} \in \mathcal{I}_{C} \cap \mathcal{I}_{C^{*}}=I\left(\right.$ alt. $(f+I) \overline{\tilde{\tau}(h+I)} \in\left(\mathcal{I}_{C_{1}} \oplus \mathcal{I}_{C_{2}}\right) \cap\left(\mathcal{I}_{C_{1}^{*}} \oplus \mathcal{I}_{C_{2}^{*}}\right)=$ I), i.e., $(f+I, h+I)=I$. Therefore

$$
\left(\mathcal{D}_{C}\right)^{\perp}=\left(\left(\mathcal{D}_{C}\right)^{\perp} \cap \mathcal{I}_{C^{\prime}}\right) \oplus \bigoplus_{C^{\prime} \neq D \in \mathcal{C}_{2}^{o}} \mathcal{I}_{D} \oplus \bigoplus_{D \in \mathcal{C}_{2}^{e}}\left(\mathcal{I}_{D_{1}} \oplus \mathcal{I}_{D_{2}}\right)
$$

(alt. $\left.\left(\mathcal{D}_{C}\right)^{\perp}=\left(\left(\mathcal{D}_{C}\right)^{\perp} \cap\left(\mathcal{I}_{C_{1}^{\prime}} \oplus \mathcal{I}_{C_{2}^{\prime}}\right)\right) \oplus \bigoplus_{D \in \mathcal{C}_{2}^{o}} \mathcal{I}_{D} \oplus \bigoplus_{C^{\prime} \neq D \in \mathcal{C}_{2}^{e}}\left(\mathcal{I}_{C_{1}^{\prime}} \oplus \mathcal{I}_{C_{2}^{\prime}}\right)\right)$, and so

$$
\begin{gathered}
\bigoplus_{C^{\prime} \in \mathcal{C}_{2}} \mathcal{D}_{C^{\prime}}^{\perp}=\bigoplus_{C \in \mathcal{C}_{2}} \mathcal{D}_{C}^{\perp}=\mathcal{D}^{\perp}=\left(\bigoplus_{C \in \mathcal{C}_{2}} \mathcal{D}_{C}\right)^{\perp} \\
=\bigoplus_{C \in \mathcal{C}_{2}^{o}}\left(\left(\mathcal{D}_{C}\right)^{\perp} \cap \mathcal{I}_{C^{\prime}}\right) \oplus \bigoplus_{C \in \mathcal{C}_{2}^{e}}\left(\left(\mathcal{D}_{C}\right)^{\perp} \cap\left(\mathcal{I}_{C_{1}^{\prime}} \oplus \mathcal{I}_{C_{2}^{\prime}}\right)\right)
\end{gathered}
$$

so that $\mathcal{D}_{C^{\prime}}^{\perp}=\left(\mathcal{D}_{C}\right)^{\perp} \cap \mathcal{I}_{C^{\prime}}$ (alt. $\mathcal{D}_{C^{\prime}}^{\perp}=\left(\mathcal{D}_{C}\right)^{\perp} \cap\left(\mathcal{I}_{C_{1}^{\prime}} \oplus \mathcal{I}_{C_{2}^{\prime}}\right)$ ). The first two items of the proposition now easily follow, since $(\cdot, \cdot)$ is nondegenerate when restricted to $\mathcal{I}_{C} \times \mathcal{I}_{C^{\prime}}$ (alt. $\mathcal{I}_{C_{1}} \oplus \mathcal{I}_{C_{2}} \times \mathcal{I}_{C_{1}^{\prime}} \oplus \mathcal{I}_{C_{2}^{\prime}}$ ), where as the first part of the third item is a consequence of equation (2).

In the case $(a)$ it is straightforward to check that

$(f+I, \overline{\tilde{\tau}(g+I)})=(f+I)(g+I)+\tilde{\tau}((f+I)(g+I))=\left(e_{C}+I\right)+\tilde{\tau}\left(\left(e_{C}+I\right)\right)=I$ so that ${ }_{S}(\overline{\tilde{\tau}(g+I)}) \subseteq \mathcal{O}(f+I)$. The fact that $(\cdot, \cdot)$ is nondegnerate implies the claimed equality, because both sets are 1 -dimensional $\mathcal{K}_{C}$-vector subspaces.

In the case $(b)$, if $f_{i}+I \neq I(i=1,2)$, then

$$
(f+I) \overline{\tilde{\tau}\left(\overline{\tilde{\tau}\left(g_{1}+g_{2}+I\right)}\right)}=\left(f_{1}+f_{2}+I\right)\left(g_{1}+g_{2}+I\right)=e_{C_{1}}+e_{C_{2}}+I \in S
$$

and so $\left(f+I, \overline{\tilde{\tau}\left(g_{1}+g_{2}+I\right)}\right)=I$, i.e., ${ }_{S}\left(\overline{\tilde{\tau}\left(g_{1}+g_{2}+I\right)}\right) \subseteq \mathcal{O}(f+I)$. Again, the fact that $(\cdot, \cdot)$ is nondegenerate implies the claimed equality. Finally, if $f_{i}+I \neq I$ (and $\left.f_{3-i}+I=I\right)$, then

$$
\left.(f+I)\left(\overline{\tilde{\tau}\left(e_{C_{3-i}^{\prime}}+I\right.}\right)\right)=\left(f_{i}+I\right)\left(e_{C_{3-i}}+I\right)=I \in S
$$

and so ${ }_{S}\left(e_{C_{3-i}^{\prime}}+I\right) \subseteq \mathcal{O}(f+I)$, if $C \neq C^{\prime}$ or $\overline{\mathcal{I}_{C_{1}}} \neq \mathcal{I}_{C_{1}}$. The other content follows from the same argument as above. The case when $C=C^{\prime}$ and $\overline{\mathcal{I}_{C_{1}}}=\mathcal{I}_{C_{1}}$ is similar.

Remark 7 Notice that, in the case $3 \mathrm{~b}$ of the previous lemma, if $f_{1}+I \neq I$, we can take $g_{1}+I \in \mathcal{I}_{C_{1}}$ the multiplicative inverse of $f_{1}+I$ in the field $\mathcal{I}_{C_{1}}$, and multiply $\left(f_{1}+f_{2}+I\right)\left(g_{1}+\tau\left(g_{1}\right)+I\right)=e_{C_{1}}+h_{2}+I$, and so $S_{S}\left(f_{1}+f_{2}+I\right)={ }_{S}\left(e_{C_{1}}+h_{2}+I\right)$. The same argument applies if $f_{2}+I \neq I$.

Example 4 (Example 1 cont'd) Let us construct the orthogonal code of the $\mathcal{D}$ presented in the example above. We first list the correspondence between 2 -classes: $\{(1,1)\}$ and $C_{2}((1, \omega))$ are fixed points in the permutation $C \leftrightarrow C^{\prime}$. On the other hand, $C_{2}((\mu, 1)) \leftrightarrow$ $C_{2}\left(\left(\mu^{6}, 1\right)\right)$ and $C_{2}((\mu, \omega)) \leftrightarrow C_{2}\left(\left(\mu^{6}, \omega\right)\right)$ Now, let us describe for each component $\mathcal{D}_{C}$, the corresponding component $\mathcal{D}_{C^{\prime}}^{\perp}$, according to Table 1.

- Since $\mathcal{D}_{\{(1,1)\}}={ }_{S}\left[\begin{array}{lllllll}1 & 1 & 1 & 1 & 1 & 1 & 1 \\ 1 & 1 & 1 & 1 & 1 & 1 & 1 \\ 1 & 1 & 1 & 1 & 1 & 1 & 1\end{array}\right]$ and $\left(1+X_{1}+X_{1}^{2}+X_{1}^{3}+X_{1}^{4}+X_{1}^{5}+X_{1}^{6}\right)(1+$ $\left.X_{2}+X_{2}^{2}\right)+I$ is the identity of $\mathcal{I}_{\{(1,1)\}}$, which happens to be invariant under $\overline{\tilde{\tau}}$, we get that $\mathcal{D}_{\{(1,1)\}}^{\perp}=\mathcal{D}_{\{(1,1)\}}$. 
- We have that $\mathcal{D}_{C_{2}((\mu, 1))}={ }_{S}\left[\begin{array}{lllllll}0 & 1 & \omega & \omega & \bar{\omega} & 1 & \bar{\omega} \\ 0 & 1 & \omega & \omega & \bar{\omega} & 1 & \bar{\omega} \\ 0 & 1 & \omega & \omega & \bar{\omega} & 1 & \bar{\omega}\end{array}\right]$, and that the inverse of the element $\left(X_{1}+\omega X_{1}^{2}+\omega X_{1}^{3}+\omega^{2} X_{1}^{4}+X_{1}^{5}+\omega^{2} X_{1}^{6}\right)\left(1+X_{2}+X_{2}^{2}\right)+I$ in the field $\mathcal{I}_{C_{2}((\mu, 1))}$ is equal to $\left(X_{1}+\omega^{2} X_{1}+\omega^{2} X_{1}^{2}+\omega^{2} X_{1}^{3}+\omega X_{1}^{4}+X_{1}^{5}+\omega X_{1}^{6}\right)\left(1+X_{2}+X_{2}^{2}\right)+I$. Therefore, $\mathcal{D}_{C_{2}((\mu, 1))^{\prime}}^{\perp}$ is generated by $\left(\omega^{2} X_{1}+X_{1}^{2}+\omega^{2} X_{1}^{3}+\omega X_{1}^{4}+\omega X_{1}^{5}+X_{1}^{6}\right)\left(1+X_{2}+X_{2}^{2}\right)$, and so $\mathcal{D}_{C_{2}((\mu, 1))^{\prime}}^{\perp}=\mathcal{D}_{C_{2}\left(\left(\mu^{6}, 1\right)\right)}$. As a consequence

$$
\mathcal{D}_{C_{2}\left(\left(\mu^{6}, 1\right)\right)^{\prime}}^{\perp}=\mathcal{D}_{C_{2}((\mu, 1))} .
$$

- From the trivial component $\mathcal{D}_{C_{2}((1, \omega))}=\left\{\left[\begin{array}{lllllll}0 & 0 & 0 & 0 & 0 & 0 & 0 \\ 0 & 0 & 0 & 0 & 0 & 0 & 0 \\ 0 & 0 & 0 & 0 & 0 & 0 & 0\end{array}\right]\right\}$ we get that

$$
\mathcal{D}_{C_{2}((1, \omega))^{\prime}}^{\perp}=\mathcal{I}_{C_{4}((1, \omega)} \oplus \mathcal{I}_{C_{4}\left(\left(1, \omega^{2}\right)\right.} .
$$

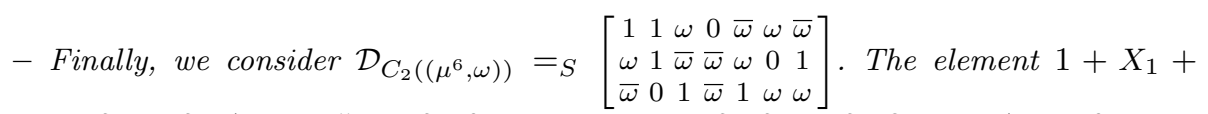
$\omega X_{1}^{2}+\omega^{2} X_{1}^{4}+\omega X_{1}^{5}+\omega^{2} X_{1}^{6}+\left(\omega+X_{1}+\omega^{2} X_{1}^{2}+\omega^{2} X_{1}^{3}+\omega X_{1}^{4}+X_{1}^{6}\right) X_{2}+$ $\left(\omega^{2}+X_{1}^{2}+\omega^{2} X_{1}^{3}+X_{1}^{4}+\omega X_{1}^{5}+\omega X_{1}^{6}\right) X_{2}^{2}+I$ decomposes as $f_{1}+f_{2}+I$, where $f_{1}+I=\left(X_{1}+X_{1}^{3}+X_{1}^{4}+X_{1}^{5}\right)\left(\omega^{2}+\omega X_{2}+X_{2}^{2}\right) \in \mathcal{I}_{C_{4}\left(\left(\mu^{6}, \omega\right)\right)}$, and $f_{2}+I=$

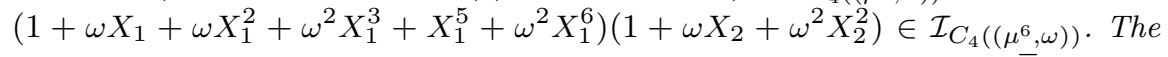
image of the sum of the inverses of $f_{1}+I$ and $f_{2}+I$ under the map $\overline{\tilde{\tau}}$ gives us $\mathcal{D}_{C_{2}\left(\left(\mu^{6}, \omega\right)\right)^{\prime}}^{\perp}=S\left[\begin{array}{ccccccc}1 & 1 & 0 & 1 & 0 & 0 & 1 \\ 1 & \omega & \bar{\omega} & \omega & 0 & \bar{\omega} & 1 \\ 0 & \bar{\omega} & \bar{\omega} & \bar{\omega} & 0 & \bar{\omega} & 0\end{array}\right]=\mathcal{D}_{C_{2}((\mu, \omega)) \text {. As a consequence }}$

$$
\mathcal{D}_{C_{2}\left(\left(\mu^{6}, \omega\right)\right)^{\prime}}^{\perp}=\mathcal{D}_{C_{2}\left(\left(\mu, \omega^{2}\right)\right)} .
$$

Therefore, the orthogonal of $\mathcal{D}$ is the additive code

$$
\begin{aligned}
\mathcal{D}^{\perp}= & \mathcal{D}_{\{(1,1)\}} \oplus \mathcal{D}_{C_{2}((\mu, 1))} \oplus \mathcal{D}_{C_{2}\left(\left(\mu^{6}, 1\right)\right)} \oplus \mathcal{I}_{C_{4}((1, \omega)} \oplus \mathcal{I}_{C_{4}\left(\left(1, \omega^{2}\right)\right.} \\
& \oplus \mathcal{D}_{C_{2}\left(\left(\mu^{6}, \omega\right)\right)} \oplus D_{C_{2}\left(\left(\mu^{6}, \omega\right)\right)}=\mathcal{D} \oplus \mathcal{I}_{C_{4}((1, \omega)} \oplus \mathcal{I}_{C_{4}\left(\left(1, \omega^{2}\right)\right.} \supseteq \mathcal{D},
\end{aligned}
$$

and so the code is self-orthogonal.

\section{Self-orthogonal and self-dual abelian codes}

Lemma 4 Let $\mathcal{D}=\bigoplus_{C \in \mathcal{C}_{2}} \mathcal{D}_{C}$ be an additive abelian code in $\mathcal{A}_{4}$ and let $\mathcal{D}^{\perp}=$ $\bigoplus_{C \in \mathcal{C}_{2}} \mathcal{D}_{C}^{\perp}$ be its dual code. Then:

1. $\mathcal{D}$ is self-orthogonal if and only if for each $C \in \mathcal{C}_{2}$, if $\mathcal{D}_{C} \neq 0$ and $\mathcal{D}_{C^{\prime}} \neq 0$, then $\mathcal{D}_{C}={ }_{S}(f+I)$ and $\mathcal{D}_{C^{\prime}}=\mathcal{O}(f+I)$, with $I \neq f+I$.

2. $\mathcal{D}$ is self-dual if and only if it is self-orthogonal and for each $C \in \mathcal{C}_{2}$ such that $\mathcal{D}_{C}=$ 0, then $\mathcal{D}_{C^{\prime}}=\mathcal{I}_{C^{\prime}}$, when $C \in \mathcal{C}_{2}^{o}\left(\right.$ or $\mathcal{D}_{C^{\prime}}=\mathcal{I}_{C_{1}^{\prime}} \oplus \mathcal{I}_{C_{2}^{\prime}}$, when $C=C_{1} \cup C_{2} \in \mathcal{C}_{2}^{e}$ ).

Proof 1. First of all notice that, for a code $\mathcal{D}$ the condition of self-orthogonality, $\mathcal{D} \subseteq$ $\mathcal{D}^{\perp}$ is equivalent to $\mathcal{D}_{C} \subseteq \mathcal{D}_{C}^{\perp}$ for all $C \in \mathcal{C}_{2}$. This condition clearly holds for all $C \in \mathcal{C}_{2}$ such that $\mathcal{D}_{C}=0$. If $\mathcal{D}_{C} \neq 0$ but $\mathcal{D}_{C^{\prime}}=0$, then $\mathcal{D}_{C}^{\perp}=\mathcal{I}_{C}$, when $C \in \mathcal{C}_{2}^{o}$, according to Theorem 3, and so the condition also holds. Finally, if $\mathcal{D}_{C} \neq 0$ and $\mathcal{D}_{C^{\prime}} \neq 0$, then $\mathcal{D}_{C} \neq \mathcal{I}_{C}$ for the code to be self-orthogonal (since otherwise, 
from Theorem $\left.3, \mathcal{D}_{C^{\prime}}^{\perp}=0 \nsupseteq \mathcal{D}_{C^{\prime}}\right)$ and $\mathcal{D}_{C^{\prime}} \neq \mathcal{I}_{C^{\prime}}$ by the same argument. Hence $\mathcal{D}_{C}={ }_{S}(f+I)$ with $I \neq f+I$. Because $\mathcal{D}_{C^{\prime}} \subseteq \mathcal{O}(f+I)$ and both sets are 1-dimensional $\mathcal{K}_{C}$ subspaces, we obtain the desired equality.

2. The code $\mathcal{D}$ is self-dual if and only if $\mathcal{D}_{C}=\mathcal{D}_{C}^{\perp}$ for all $C \in \mathcal{C}_{2}$. In particular it has to be self-orthogonal. For the classes $C \in \mathcal{C}_{2}$ such that $\mathcal{D}_{C}={ }_{S}(f+I)$ and $\mathcal{D}_{C^{\prime}}=\mathcal{O}(f+I)$ (with $I \neq f+I$ ) the equality is true, whereas if $0=\mathcal{D}_{C}=\mathcal{D}_{C}^{\perp}$, from Theorem 3, we must have $\mathcal{D}_{C^{\prime}}=I_{C^{\prime}}$, for the code to be self-dual. The case $C \in \mathcal{C}_{2}^{e}$ is similar. in $\mathcal{A}_{4}$.

Now, we are able to describe the self-orthogonal and self-dual additive abelian codes

Theorem 4 Let $\mathcal{D}=\bigoplus_{C \in \mathcal{C}_{2}} \mathcal{D}_{C}$ be an additive abelian code in $\mathcal{A}_{4}$ and let $\mathcal{D}^{\perp}=$ $\bigoplus_{C \in \mathcal{C}_{2}} \mathcal{D}_{C}^{\perp}$ be its dual code. Then, $\mathcal{D}$ is self-orthogonal (alt. $\mathcal{D}$ is self-dual) if and only if for each $C \in \mathcal{C}_{2}$ the following condition hold (alt. except those marked with an asterisk):

1. If $C \in \mathcal{C}_{2}^{o}$ :

(a) If $C=C_{2}((1, \ldots, 1))$, then either

$-\mathcal{D}_{C}=\{0\}(*)$

- or $\mathcal{D}_{C}$ is any $\mathcal{K}_{C}$-vector space of dimension 1.

(b) In other case, either

i. $\mathcal{D}_{C}=\{0\}$ and

$-\mathcal{D}_{C^{\prime}}=\mathcal{I}_{C^{\prime}}$

- or $\mathcal{D}_{C^{\prime}}$ is any other $\mathcal{K}_{C^{\prime} \text {-vector subspace of } \mathcal{I}_{C^{\prime}}(*)}$

ii. or $\mathcal{D}_{C}={ }_{S}(f+I)$ with $f+I \neq I$, and $\mathcal{D}_{C^{\prime}}={ }_{S}(\tilde{\tau}(g+I))$, where $g+I \in \mathcal{I}_{C}$ is the multiplicative inverse of $f+I$ in the field $\mathcal{I}_{C}$.

2. If $C=C_{1} \cup C_{2} \in \mathcal{C}_{2}^{e}$ :

(a) If $C=C^{\prime}$, then either

i. $\mathcal{D}_{C}=\{0\}(*)$,

ii. or if $\overline{\mathcal{I}_{C_{i}}}=\mathcal{I}_{C_{i}}(i=1,2)$, then

- $\mathcal{D}_{C}={ }_{S}\left(e_{C_{i}}+I\right)$ with $i=1,2$

- or $\mathcal{D}_{C}=_{S}\left(f_{1}+\tilde{\tau}\left(f_{1}\right) z+I\right)$ where $z+I$ is a non-zero element of the subfield of $\mathcal{I}_{C_{2}}$ isomorphic to $\mathbb{F}_{2^{\left|C_{1}\right|}}$

iii. or if $\overline{\mathcal{I}_{C_{1}}}=\mathcal{I}_{C_{2}}$, then $\mathcal{D}_{C}={ }_{S}\left(f_{1}+\tilde{\tau}\left(f_{1}\right) z+I\right)$ where $z+I$ is a non-zero element of $\mathcal{I}_{C_{2}}$ of order $2^{\left|C_{1}\right|}+1$.

(b) If $C \neq C^{\prime}$, then either

i. $\mathcal{D}_{C}=\{0\}$ and

$-\mathcal{D}_{C^{\prime}}=\mathcal{I}_{C^{\prime}}$

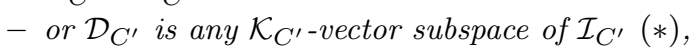

ii. or $\mathcal{D}_{C}={ }_{S}\left(e_{C_{i}}+I\right)(i=1,2)$ and $\mathcal{D}_{C^{\prime}}={ }_{S}\left(e_{C_{i}^{\prime}}+I\right)$,

iii. or $\mathcal{D}_{C}=_{S}\left(f_{1}+f_{2}+I\right)$ with $f_{i}+I \neq I$ for $i=1,2$, and $\mathcal{D}_{C^{\prime}}={ }_{S}$ $\left(\overline{\tilde{\tau}\left(g_{1}+g_{2}+I\right)}\right)$, where $g_{i}+I \in \mathcal{I}_{C_{i}}$ is the multiplicative inverse of $f_{i}+I$ in the field $\mathcal{I}_{C_{i}}(i=1,2)$.

These possibilities for self-orthogonal (alt. self-dual) additive abelian codes are summarized in Table 2.

Proof 1. (a) If $C=C_{2}((1, \ldots, 1))$, then $C=C^{\prime}$ and so, for the code to be selforthogonal $\mathcal{D}_{C}=\mathcal{D}_{C^{\prime}}$ must be a proper $\mathcal{K}_{C}$-vector subspace of $\mathcal{I}_{C}$ (according 


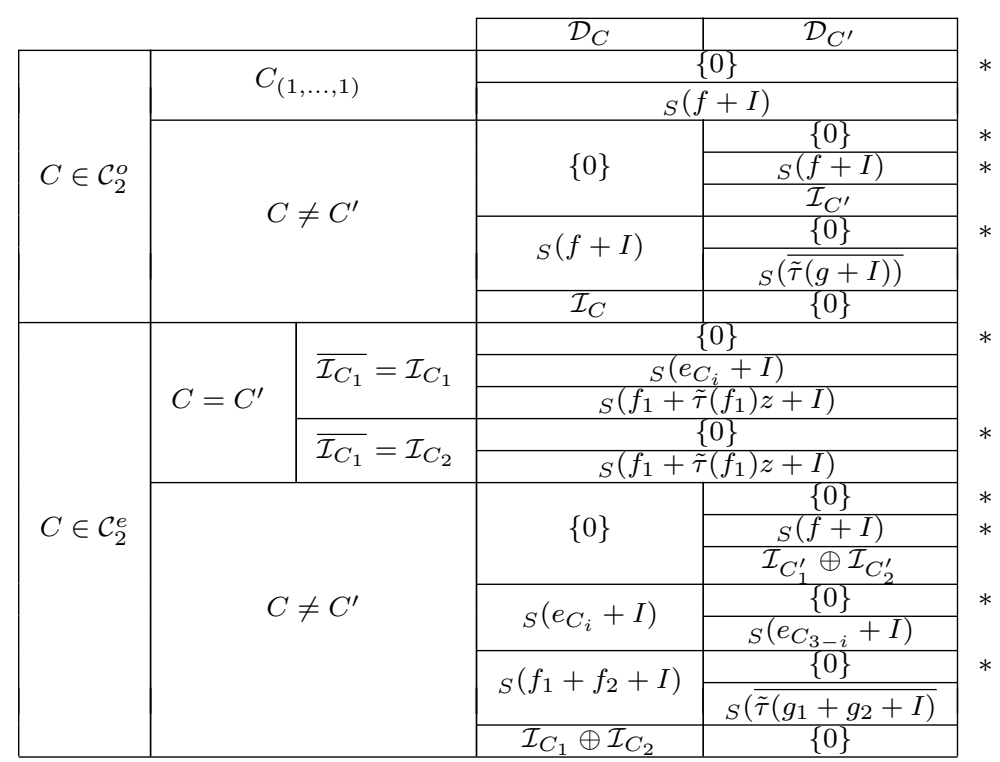

Table 2 Different possibilities on the summands for a code to be self-orthogonal or self-dual

to Lemma 4.1). The case $\mathcal{D}_{C}=\mathcal{D}_{C^{\prime}}=\{0\}$ is allowed for self-orthogonality, but it is not acceptable for self-duality (according to Lemma 4.2). Finally, if $\mathcal{D}_{C}={ }_{S}(f+I)$, from Theorem 3 we know that $\mathcal{D} \stackrel{\perp}{C}=\mathcal{O}(f+I)={ }_{S}(\overline{\tilde{\tau}(g+I)})$, where $g+I$ is the multiplicative inverse of $f+I$ in the field $\mathcal{I}_{C}$. Thus, ${ }_{S}(f+$ $I)=_{S}(\overline{\tilde{\tau}(g+I)})$ if and only if there exists $c+I \in S$ such that $(c+I)(f+I)=$ $\overline{\tilde{\tau}(g+I)}$, if and only if $c+I=\overline{\tilde{\tau}(g+I)}(g+I) \in S$. The restriction of the ring automorphism $:$ to the ideal $\mathcal{I}_{C}$ is the identity, and so $c+I=\tilde{\tau}(g+I)(g+I)=$ $\tilde{\tau}(c+I)$, i.e., $c+I \in S$ always.

(b) In this case $C \neq C^{\prime}$, and so self-orthogonality in the subcase i. (i.e., $\mathcal{D}_{C}=\{0\}$ ) is obvious (but self-duality is not possible unless $\mathcal{D}_{C^{\prime}}=\mathcal{I}_{C^{\prime}}$ ), according to Lemma 3. In the subcase ii. $\left(\mathcal{D}_{C}={ }_{S}(f+I)\right)$ self-orthogonality is only true when $\mathcal{D}_{C^{\prime}}={ }_{S}(\overline{\tilde{\tau}(g+I)})$, because of Theorem 3. Since $\tilde{\tau}$ and - are commuting ring automorphisms of order at most 2, it follows that $\mathcal{D}_{C}=_{S}(f+I)=_{S}$ $(\overline{\tilde{\tau}(\overline{\tilde{\tau}(f+I)})})=\mathcal{O}(\overline{\tilde{\tau}(g+I)})$ and thus the condition is also sufficient. Selfduality is always true in such a case.

2. (a) If $C=C^{\prime}=C_{1} \cup C_{2}$, the case $\mathcal{D}_{C}=\{0\}$ is possible if $\mathcal{D}$ is self-orthogonal, but not if it is self-dual (Lemma 4).

If $C \in \mathcal{C}_{2}$, and $\overline{\mathcal{I}_{C}}=\mathcal{I}_{C}$, then the restriction of $:$ to $\mathcal{I}_{C}$ is the identity if and only if $C=C_{2}((1, \ldots, 1))$. In fact, in these conditions, if $f+I=\sum_{\alpha} f_{\alpha} X^{\alpha}+I \in$ $\mathcal{I}_{C}$, then $\overline{X^{\beta}(f+I)}=X^{\beta}(f+I)$ for any index $\beta=\left(\beta_{1}, \ldots, \beta_{r}\right)$. So, this implies that $f_{n-\alpha-\beta}=f_{\alpha-\beta}$ for all $\alpha$ and $\beta$. Taking $\alpha=\beta$ we have that $f_{n-2 \beta}=f_{0}$ for all $\beta$. Since $n_{i}$ is odd for $i=1, \ldots, r, f+I=f_{0}\left(\sum_{\alpha} X^{\alpha}+I\right)$ which is an element of $\mathcal{I}_{C}$ with $C=C_{2}((1, \ldots, 1))$.

Let us suppose that $\overline{\mathcal{I}_{C_{i}}}=\mathcal{I}_{C_{i}}$ for $i=1,2$. Hence, $\overline{\mathcal{I}_{C}}=\mathcal{I}_{C}$ and the restriction $\left.{ }^{-}\right|_{\mathcal{I}_{C_{i}}}$ is a non trivial field automorphism or order 2, i.e., $\overline{f_{i}+I}=f_{i}^{2^{d}}+I$, 
where $d=\left|C_{i}\right|$, since $\mathcal{I}_{C_{i}} \cong \mathbb{F}_{4^{\left|C_{i}\right|}}$. If $\mathcal{D}_{C}={ }_{S}\left(f_{i}+I\right) \quad(i=1$ or 2$)$, then $\mathcal{O}\left(f_{i}+I\right)={ }_{S}\left(e_{C_{i}}+I\right)$, and so self-orthogonality implies ${ }_{S}\left(f_{i}+I\right)={ }_{S}\left(e_{C_{i}}+I\right)$. In such a case $\mathcal{D}_{C}=\mathcal{D}_{C^{\prime}}$, and the condition for self-duality if also satisfied. Otherwise, $\mathcal{D}_{C}={ }_{S}\left(f_{1}+f_{2}+I\right)$ with $f_{i}+I \neq I(i=1,2)$, and $\mathcal{O}\left(f_{1}+f_{2}+I\right)=_{S}$ $\left(\overline{\tilde{\tau}\left(g_{1}+g_{2}+I\right)}\right.$, where $g_{i}+I$ is the multiplicative inverse of $f_{i}+I$ in the field $\mathcal{I}_{C_{i}}$. Therefore, the condition for self-orthogonality (and self-duality) holds if and only if there exists an element $c+I \in S$ such that

$$
f_{1}+f_{2}+I=(c+I)\left(\overline{\tilde{\tau}\left(g_{1}\right)}+\overline{\tilde{\tau}\left(g_{2}\right)}+I\right)=(c+I)\left(\tilde{\tau}\left(g_{1}\right)^{2^{d}}+\tilde{\tau}\left(g_{2}\right)^{2^{d}}+I\right)
$$

Then, the element $c+I \in S$ must verify the following condition

$$
c+I=\left(f_{1}+f_{2}+I\right)\left(\tilde{\tau}\left(f_{1}\right)^{2^{d}}+\tilde{\tau}\left(f_{2}\right)^{2^{d}}+I\right)=f_{1} \tilde{\tau}\left(f_{2}\right)^{2^{d}}+f_{2} \tilde{\tau}\left(f_{1}\right)^{2^{f}}+I
$$

Now, since $\tilde{\tau}(c+I)=c+I$, we arrive to these equations

$$
\left\{\begin{array}{l}
f_{1} \tilde{\tau}\left(f_{2}\right)^{2^{d}}+\tilde{\tau}\left(f_{2}\right) f_{1}^{2^{d}}+I=I \\
f_{2} \tilde{\tau}\left(f_{1}\right)^{2^{d}}+\tilde{\tau}\left(f_{1}\right) f_{2}^{2^{d}}+I=I
\end{array}\right.
$$

From the second equation, we obtain that $\left.\left(\tilde{\tau}\left(f_{1}\right) g_{2}\right)^{2^{d}-1}+I\right)=e_{C_{2}}+I$, that is, $\tilde{\tau}\left(f_{1}\right) g_{2}+I$ is a non-zero element of the subfield of $\mathcal{I}_{C_{2}}$ isomorphic to $\mathbb{F}_{2^{d}}$. So, we obtain $f_{2}+I=\tilde{\tau}\left(f_{1}\right) z+I$. This solution also verifies the first equation, so the result follows.

Let us suppose that $\overline{\mathcal{I}_{C_{1}}}=\mathcal{I}_{C_{2}}$. If $\mathcal{D}_{C}={ }_{S}\left(f_{i}+I\right)$ with $i=1,2, \mathcal{O}\left(f_{i}+I\right)=_{S}$ $\left(e_{C_{3-i}}+I\right) \neq \mathcal{D}_{C}$, and so the code can not be self-orthogonal. Finally, we will consider $\mathcal{D}_{C}={ }_{S}\left(f_{1}+f_{2}+I\right)$. Since the restriction $\left.\overline{\tilde{\tau}}\right|_{\mathcal{I}_{C_{1}}}\left(\right.$ alt. $\left.\left.\overline{\tilde{\tau}}\right|_{\mathcal{I}_{C_{2}}}\right)$ is a field automorphism of order 2, i.e., $\overline{\tilde{\tau}\left(f_{i}+I\right)}=f_{i}^{2^{d}}+I$, using the same argument of the case $2($ a)ii, we arrive to the equations

$$
\left\{\begin{array}{l}
f_{1}^{2^{d}+1}+\tilde{\tau}\left(f_{2}\right)^{2^{d}+1}+I=I \\
f_{2}^{2^{d}+1}+\tilde{\tau}\left(f_{1}\right)^{2^{d}+1}+I=I .
\end{array}\right.
$$

Solving the second equation we obtain $f_{2}+I=\tilde{\tau}\left(f_{1}\right) z+I$, where $z+I$ is a non-zero element of $\mathcal{I}_{C_{2}}$ of order $2^{d}+1$. This solution also satisfies the first equation, and the result follows.

(b) Let us now suppose that $C \neq C^{\prime}$. The first case is similar to $1(b) i$, where as the second one is similar to the first subcase of $2(a)$ ii. Finally, if $\mathcal{D}_{C}={ }_{S}\left(f_{1}+f_{2}+I\right)$ with $f_{i}+I \neq I$ for $i=1,2$, then $\left.\mathcal{O}\left(f_{1}+f_{2}+I\right)=_{S}\left(\overline{\tilde{\tau}\left(g_{1}+g_{2}+I\right.}\right)\right)$, where $g_{i}+I$ is the multiplicative inverse of $f_{i}+I(i=1,2)$. Hence, $\mathcal{D}_{C^{\prime}}=_{S}\left(\overline{\tilde{\tau}\left(g_{1}+g_{2}+I\right)}\right)$ for the code to be self-orthogonal and self-dual.

Example 5 (Example 1 cont'd) The components of the self-orthogonal code $\mathcal{D}$ presented in the examples above fall in the following cases of Theorem 4:

- $\mathcal{D}_{\{(1,1)\}}$ is case $1(a)$,

- $\mathcal{D}_{C_{2}((\mu, 1))}$ and $\mathcal{D}_{C_{2}\left(\left(\mu^{6}, 1\right)\right)}$ are case $1(b) i i$,

- $\mathcal{D}_{C_{2}((1, \omega))}$ is case $2(a) i$,

- and $\mathcal{D}_{C_{2}\left(\left(\mu, \omega^{2}\right)\right)}$ and $\mathcal{D}_{C_{2}\left(\left(\mu^{6}, \omega\right)\right)}$ are case $2(b)$ iii. 
Let us observe that this self-orthogonal code $\mathcal{D}$ allow us to construct, via [2][Theorem 2], a quantum-error-correcting code with parameters $[[21,2, d]]$, where $d$ is the smallest weight of codewords in $\mathcal{D}^{\perp} \backslash \mathcal{D}$. Sage computations [16] show that the actual distance of the quantum-error-correcting code is $d=6$, i.e., it has the same distance as the best known code with the same parameters listed in [6].

The ring automorphism $\overline{\tilde{\tau}}$ defines an equivalence relation over the set of 2-classes $\mathcal{C}_{2}$. Let us denote $\mathcal{B}$ the quotient set of $\mathcal{C}_{2}$ by this relation. Using this notation, the number of self-orthogonal and self-dual codes are given by the following result.

Corollary 2 The number of additive abelian self-orthogonal (alt. self-dual) codes is given by Table 3.

\begin{tabular}{|l|c|c|}
\cline { 2 - 4 } \multicolumn{1}{c|}{ Any } & Generated by a single word \\
\hline Self-orthogonal & $4 \prod_{\substack{C \in \mathcal{B} \\
C \neq C^{\prime}}}\left(3 \cdot 2^{|C|}+6\right) \prod_{\substack{C \in \mathcal{B} \\
C=C^{\prime}}}\left(2^{\frac{|C|}{2}}+2\right)$ & $4 \prod_{\substack{C \in \mathcal{B} \\
C \neq C^{\prime}}}\left(3 \cdot 2^{|C|}+4\right) \prod_{\substack{C \in \mathcal{B} \\
C=C^{\prime}}}\left(2^{\frac{|C|}{2}}+2\right)$ \\
\hline Self-dual & $3 \prod_{\substack{C \in \mathcal{B} \\
C \neq C^{\prime}}}\left(2^{|C|}+3\right) \prod_{\substack{C \in \mathcal{B} \\
C=C^{\prime}}}\left(2^{\frac{|C|}{2}}+1\right)$ & $3 \prod_{\substack{C \in \mathcal{B} \\
C \neq C^{\prime}}}\left(2^{|C|}+1\right) \prod_{\substack{C \in \mathcal{B} \\
C=C^{\prime}}}\left(2^{\frac{|C|}{2}}+1\right)$ \\
\hline
\end{tabular}

Table 3 Number of self-orthogonal and self-dual codes

Proof In Table 4 we count the number of codes from the possible choices in the entries of the table of Theorem 4 (see also Remark 7).

In order to know the number of self-dual codes, we do no count rows marked with an asterisk. Finally, the code $\mathcal{D}$ is generated by a single word if and only if $\mathcal{D}_{C}$ is a $\mathcal{K}_{C}$-vector space of dimension 0 or 1 for all $C \in \mathcal{C}_{2}$, i.e., we do not count any row containing $\mathcal{I}_{C}$ or $\mathcal{I}_{C_{1}} \oplus \mathcal{I}_{C_{2}}$.

Acknowledgements Edgar Martínez-Moro was partially funded by Spanish MCINN under projects MTM2007-64704 and MTM2010-21580-C02-02. A. Piñera-Nicolás and I. F. Rua were supported by MTM2010-18370-C04-01. 


\begin{tabular}{|c|c|c|c|c|c|c|}
\hline & & & & \\
\hline & & & $\mathcal{D}_{C}$ & $\mathcal{D}_{C^{\prime}}$ & \multirow{2}{*}{$\begin{array}{c}\text { Total number } \\
4\end{array}$} & \multirow{7}{*}{$3 \cdot 2^{|C|}+6$} \\
\hline \multirow{6}{*}{$C \in \mathcal{C}_{2}^{o}$} & \multicolumn{2}{|c|}{$C_{(1, \ldots, 1)}$} & \multicolumn{2}{|c|}{$\frac{1}{\frac{2^{2}-1}{2-1}}$} & & \\
\hline & \multirow{5}{*}{\multicolumn{2}{|c|}{$C \neq C^{\prime}$}} & \multirow{3}{*}{1} & \multirow{3}{*}{$\begin{array}{c}1 \\
\frac{2^{|C|}-1}{2-1} \\
1\end{array}$} & \multirow{3}{*}{$2^{|C|}+3$} & \\
\hline & & & & & & \\
\hline & & & & & & \\
\hline & & & $\frac{2^{|C|}-1}{2-1}$ & $\frac{1}{1}$ & $\left(2^{|C|}+1\right) \cdot 2$ & \\
\hline & & & 1 & 1 & 1 & \\
\hline \multirow{8}{*}{$C \in \mathcal{C}_{2}^{e}$} & \multirow{3}{*}{$C=C^{\prime}$} & \multirow{2}{*}{$\overline{\mathcal{I}_{C_{1}}}=\mathcal{I}_{C_{1}}$} & 2 & $\begin{array}{l}1 \\
-1\end{array}$ & \multirow{2}{*}{$2^{|C| / 2}+2$} & \multirow{3}{*}{$2^{|C| / 2}+2$} \\
\hline & & & \multicolumn{2}{|c|}{$2^{|C| / 2}-1$} & & \\
\hline & & $\overline{\mathcal{I}_{C_{1}}}=\mathcal{I}_{C_{2}}$ & \multicolumn{2}{|c|}{1} & $2^{|C| / 2}+2$ & \\
\hline & \multirow{5}{*}{\multicolumn{2}{|c|}{$C \neq C^{\prime}$}} & 1 & $\frac{1}{\frac{2^{|C|}-1}{2-1}} \frac{1}{1}$ & $2^{|C|}+3$ & \multirow{5}{*}{$3 \cdot 2^{|C|}+6$} \\
\hline & & & \multirow{2}{*}{2} & 1 & \multirow{2}{*}{4} & \\
\hline & & & & 1 & & \\
\hline & & & $2^{|C|}-1$ & $\frac{1}{1}$ & $\left(2^{|C|}-1\right) \cdot 2$ & \\
\hline & & & 1 & 1 & 1 & \\
\hline
\end{tabular}

Table 4 Total number of self-orthogonal and self-dual codes

\section{References}

1. S. D. Berman. On the theory of group codes. Cybernetics, 3(1):25-31 (1969), 1969.

2. A. Calderbank, E. Rains, P. Shor, and N. Sloane. Quantum error correction via codes over $G F(4)$. IEEE Trans. Inform. Theory, 44:1369-1387, 1998.

3. P. Charpin. Une généralisation de la construction de Berman des codes de Reed et Muller p-aires. Comm. Algebra, 16(11):2231-2246, 1988.

4. D. Cox, J. Little, and D. O'Shea. Ideals, Varieties, and Algorithms. Springer, New York, $200 \%$.

5. B. Dey and B. Rajan. $\mathbb{F}_{q}$-linear cyclic codes over $\mathbb{F}_{q}^{m}$. Des. Codes Cryptogr., 34:89-116, 2005.

6. M. Grassl. Bounds on the minimum distance of linear codes and quantum codes. Online available at http://www. codetables.de

7. W. C. Huffman. Additive cyclic codes over $\mathbb{F}_{4}$. Adv. Math. Commun., 1(4):427-459, $200 \%$.

8. W. C. Huffman. Additive cyclic codes over $\mathbb{F}_{4}$. Adv. Math. Commun., 2(3):309-343, 2008.

9. E. Martínez-Moro and I. F. Rúa. Multivariable codes over finite chain rings: serial codes. SIAM J. Discrete Math., 20(4):947-959, 2006.

10. E. Martínez-Moro and I. F. Rúa. On repeated-root multivariable codes over a finite chain ring. Des. Codes Cryptogr., 45:219-227, 2007.

11. W. W. Peterson and J. E. J. Weldon. Error-correcting codes. The M.I.T. Press, Cambridge, Mass.-London, second edition, 1972.

12. A. Poli. Important algebraic calculations for n-variables polynomial codes. Discrete Math., 56(2-3):255-263, 1985.

13. A. Poli and L. Huguet. Error correcting codes. Prentice Hall International, Hemel Hempstead, 1992.

14. P. Shor. Scheme for reducing decoherence in quantum memory. Phys. Rev. A, 52, 1995.

15. A. Steane. Simple quantum error correcting codes. Phys. Rev. Lett., 77:793-797, 1996.

16. W.A. Stein et al. Sage Mathematics Software (Version 3.0.1). The Sage Development Team, 2008. http://www. sagemath.org 